\title{
Binding Energy, Conformational Change, and the Mechanism of Transmembrane Solute Movements
}

\author{
GENE A. SCARBOROUGH \\ Department of Pharmacology, School of Medicine, University of North Carolina at Chapel Hill, Chapel Hill, North \\ Carolina 27514
}

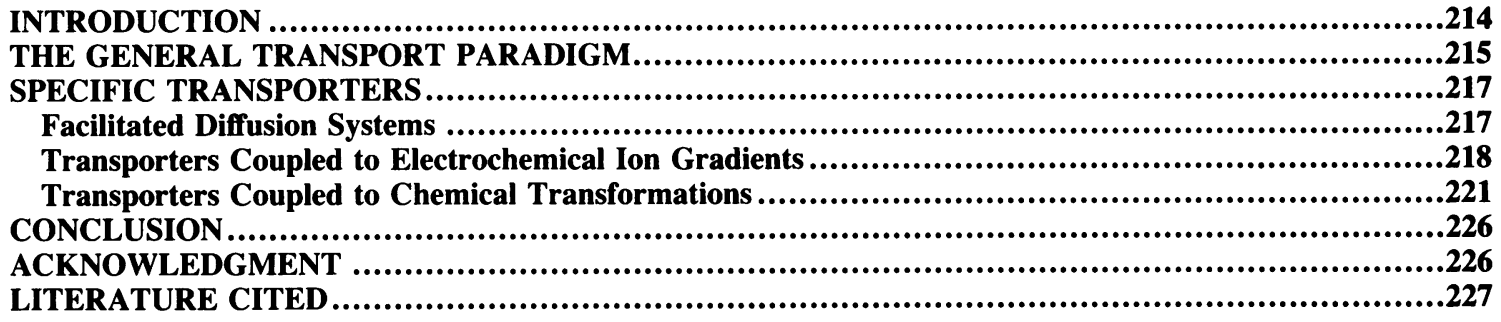

\section{INTRODUCTION}

Ever since the plasmolysis experiments of Overton (139) and his perspicacious suggestion arising therefrom that living cells are surrounded by a lecithin- and cholesterol-containing lipoid barrier, or plasma membrane as we now know it, it has been a question of general and significant interest as to how polar compounds are made able to penetrate this barrier. Moreover, as it subsequently became obvious that certain polar compounds not only readily penetrate the plasma membrane, but accumulate on one side of it, a second major question arose as to the mechanism by which energy is supplied to drive the process. Since the last turn of the century, countless experimentalists and theoreticians have been engaged by these questions, and as a result of their collective efforts, vast amounts of specific information pertinent to these two questions have been generated. The general picture that has emerged from all of this activity is that cells contain from one to several anatomically distinct membranes and that the penetration of virtually all of these membranes by polar compounds much larger than water molecules is, for the most part, catalyzed by solute-specific membrane-bound proteins, which will hereafter be referred to as transporters. Furthermore, for transporters that catalyze the transmembrane accumulation of polar solutes, it is also now clear that, depending upon the specific system in question, energy may be supplied in chemical form such as adenosine triphosphate (ATP), phosphoenolpyruvate, or oxidizable substrates, or in the form of transmembrane electrochemical gradients of ions, most commonly protons or sodium ions, or in the form of light. And, it is also now clear that, owing to the fact that the reactions catalyzed by most transporters are reversible, the above-mentioned forms of energy are, to a great extent, interconvertible in the presence of the appropriate transporter or transporters. Thus, in certain procaryotes and the chloroplasts of plants, harvested light energy is converted by electron transfer-driven proton transporters into energy in the form of a transmembrane electrochemical proton gradient, which in turn is converted into chemical energy in the form of ATP via a transporter known as the $\mathrm{F}_{1} \mathrm{~F}_{O} \mathrm{H}^{+}$-adenosine triphosphatase (ATPase)/ATP synthase. Similar cascades fueled by oxida- tion reactions occur in many procaryotes and the mitochondria of eucaryotic organisms. And, in a conceptually similar fashion, ATP-, electron transfer- and light-driven ion transporters in plasma membranes and a variety of subcellular organelles in many cell types generate transmembrane gradients of $\mathrm{H}^{+}, \mathrm{Na}^{+}, \mathrm{K}^{+}$, and $\mathrm{Ca}^{2+}$, which are in turn used by specific ion gradient-coupled transporters to drive nutrient accumulation, extrusion of certain intracellular constituents, and a variety of transmembrane signalling mechanisms as well.

The widespread acceptance of these views lends support to their general validity, and it must therefore be acknowledged that the field has made significant progress. Nevertheless, as we approach the next turn of the century, we do so with the unsettling realization that in spite of the facts that numerous transporters have been defined and biochemically characterized, and their role in cellular energy interconversion is appreciated, we still know very little about how they really work. Little progress has been made in evolving a conceptually satisfactory model for the molecular mechanism of transport, and thus major questions remain as to the events that transpire as solutes breech the membrane barrier. It is not that there is a dearth of models to choose from, for, to be sure, there are a great many. The problem is that the classical investigative tools of thermodynamics and kinetics do not independently provide information as to molecular mechanism, and in the absence of high-resolution structural information about transporter molecules, scientists intent upon understanding how transporters work are forced to rely to some extent upon their imaginations in the formulation of models to explain the transport process. There is, of course, nothing wrong with that, for imagination is usually at the root of innovative scientific discovery (122, 147). Unfortunately, for reasons that are not entirely clear, but are probably related to uncertainty as to what membrane transport proteins might really be like, imagined mechanisms for transporters almost invariably contain one or more undefined "black box" steps, which leave the reader pondering how a protein might actually accomplish them. The primary processes that usually remain unexplained are the so-called translocation step, in which binding sites occupied 
by the species to be transported somehow undergo a change in position from the uptake side to the release side of the membrane, and the so-called energy coupling step, in which energy input somehow changes the binding sites from high to low affinity as they move from the uptake side to the release side of the membrane. And, it is precisely this lack of specific information as to what the translocation and energy coupling processes might actually entail that circumscribes the present state of uncertainty in the transport field.

On several occasions in the past $(22,158,159)$, once in some detail (159), I have attempted to explain my notion, drawn largely from the writings of Peter Mitchell (e.g., 112, $114,116-118,120,121,123-125,127-129)$, well-established principles of enzymatic catalysis, and the recent virtual explosion of information in the field of protein structure and dynamics, that transport can be explained in biochemically orthodox terms without the necessity of any nonexplicit black box steps. This notion was developed within the context of the mechanisms of the ion-translocating ATPases of the aspartyl-phosphoryl-enzyme intermediate family of transporters, and the models that resulted from those considerations stressed transition state binding energy and electrovalent or covalent association or both of the transported ions with the species undergoing the chemical transformations. Further recent work along these lines has now made it clear that the principles underlying the specific details of those models are applicable to virtually all transporters, whether or not chemical transformations or ions or both are involved. In the present article, these ideas are developed into a general transport paradigm. It is then shown how the mechanisms of most of the known transporters can be viewed within this framework. The encouraging indication is that virtually all transporters could operate via a single fundamental mechanism.

\section{THE GENERAL TRANSPORT PARADIGM}

It is appropriate to begin with a few brief comments on what is presently known about the nature of the interactions of proteins with their ligands and the predominant type of conformational change that occurs as a result of these interactions. The available experimental evidence in support of protein conformational changes associated with ligand binding is extensive (e.g., 3, 16, 32, 41, 54, 55, 60, 62, 63, 77, $87,98-100,102,110,132,133,150,164)$, but possibly the most thoroughly studied case is that of the periplasmic L-arabinose-binding protein of Escherichia coli. In this case, the three-dimensional structure of the ligand-protein complex is known with high resolution $(47,132,134)$, and the thermodynamics and kinetics of the events that take place upon ligand binding are reasonably well understood (41, $98-100,110,132,133)$. The general picture that has evolved from these elegant studies is that the $\mathrm{L}$-arabinose-binding protein consists of two relatively rigid domains or lobes of roughly equal mass, separated by a cleft and connected by a relatively flexible, so-called hinge region. The arabinosebinding site comprises residues from both lobes and is not complete in the unliganded form of the protein because the thermodynamically most stable form of the unliganded protein is with the lobes poised relatively distant from one another, i.e., with the cleft open. Upon the binding of a suitable substrate such as L-arabinose, presumably to some part of the binding site, there ensues a rapid hinge-bending and cleft-closing conformational change, which completes the formation of the binding site and embeds the sugar roughly in the center of mass of the molecule, virtually inaccessible from the aqueous environment. The entire process has been colorfully likened to the mechanism of the Venus' flytrap (100) and is the embodiment of one form of Koshland's original notion of induced fit (87). The forces that drive and stabilize cleft closure are both entropic and enthalpic $(41,98,99)$, and it is also pertinent that the relative affinities of the protein for various sugars are, in large part, determined by how well the individual sugars hold the lobes together, because the association rate constants for numerous sugars are similar, whereas it is the dissociation rate constants (opening of the trap) that vary in magnitude. For example, the association rate constants for $L$-arabinose and D-fucose are roughly $2.4 \times 10^{7}$ and $1.2 \times 10^{7} \mathrm{M}^{-1} \mathrm{~s}^{-1}$, respectively, but the dissociation rate constants are about 1.5 and $37 \mathrm{~s}^{-1}$, leading to binding constants roughly 50 -fold different (110).

This general behavior is not confined to the arabinosebinding protein, nor just to bacterial periplasmic binding proteins as a class, but is also observed for a number of other systems in which proteins and ligands interact, including the immunoglobulins $(63,102)$, virus coat proteins $(54,55,63)$, and, most importantly for our present purposes, enzymes, including lysozyme (62), citrate synthase (150), alcohol dehydrogenase $(31,32)$ and other dehydrogenases $(63)$, most if not all kinases $(3,63)$, and probably enzymes in general (164). The probability that enzymes behave in this way upon substrate binding is particularly appealing because the conflicting requirements of substrate access and transition state affinity (195) are both well accommodated. That is, as suggested first by Pauling (144) and explained again by others $(65,94,194)$, enzymes have their highest affinity for the transition state configuration of the atoms involved in the reaction being catalyzed, and as elaborated upon by Wolfenden (195), this might occur with the maximum possible efficiency when the transition state is more or less totally surrounded by the enzyme. The problem with this is that substrates of any significant size could not gain access to such an active site. The hinge-bending maneuver would appear to be a perfect solution to the problem, allowing both ready substrate access and maximum transition state affinity. As an added attractive feature, after the transition state has broken down, which it does spontaneously at a great rate (40), the forces holding the cleft closed are expected to be significantly weakened, which would facilitate cleft reopening, product release, and the initiation of another catalytic cycle. By analogy with the L-arabinosebinding protein, this would be like changing L-arabinose (high affinity) to D-fucose (low affinity) while it is deep within the binding site. This key sequence of events that occurs in the process of enzymatic catalysis, i.e., a substrate-induced or stabilized (or both) hinge-bending conformational change followed by events that tend to reverse the conformational change, foreshadows a similar sequence proposed below as the central feature of the catalytic mechanisms of transporters.

The accumulation of experimental evidence for and the intuitive attractiveness of the hinge-bending motif engenders confidence that it is a fundamental biological phenomenon, at least in the case of water-soluble proteins. It remains for the present argument to discuss whether or not membrane proteins can be expected to behave in a similar manner. In the early days of membranology, our ignorance of the properties of membrane proteins and attendant inability to manipulate, isolate, and purify them led to the general notion that they are fundamentally different than soluble proteins. However, as our knowledge of membrane proteins has 
expanded owing to the experimental efforts of numerous investigators, it is now beginning to appear as if membrane proteins may not be as different from water-soluble proteins as they were once thought to be. An excellent case in point is the $\mathrm{Ca}^{2+}$-translocating ATPase of sarcoplasmic reticulum. This integral membrane protein can be rendered monomeric, water soluble, and virtually lipid-free, yet stable and fully active, when prepared as a complex with the detergent dodecyl octaethylene glycol monoether (23). Binding of the detergent appears to be a simple equilibrium phenomenon, and it can be conservatively estimated (17) that the detergent-binding region of this enzyme occupies less than onequarter of the total surface area. The rest of the surface is apparently much like more conventional soluble proteins, and the forces that stabilize that part of the enzyme are likely to be no different than the forces that stabilize soluble proteins. The simple picture that arises from these observations is that the sarcoplasmic reticulum ATPase is stable and active in aqueous solution provided that its hydrophobic sector, normally stabilized by the lipid bilayer, is stabilized by other suitable hydrophobic ligands such as those provided by the detergent dodecyl octaethylene glycol monoether. Considering membrane proteins in this way imparts a satisfying element of rationality and predictability to a field that has been historically largely empirical and encourages the expectation that most, if not all, membrane proteins will ultimately be found to behave in a similar fashion. Additional experimental support for this notion comes from the studies of Brotherus et al., who succeeded in preparing active protomers of the $\mathrm{Na}^{+} / \mathrm{K}^{+}$-ATPase $\alpha, \beta$ heterodimer (12); moreover, at least six other integral membrane proteins, including bacteriorhodopsin, matrix porin, maltoporin, conjugin, mitochondrial cytochrome $b c_{1}$ complex, and cytochrome $b_{5}$ have also been prepared as stable, monodisperse, protein-detergent complexes $(26,45,46,108$, $131,142,161,184)$. The mitochondrial $\mathrm{F}_{1} \mathrm{~F}_{O} \mathrm{H}^{+}$ATPase/ATP synthase has also recently been isolated as a highly dispersed, protein-detergent complex (103). In view of this growing body of evidence documenting the stability of membrane proteins in aqueous solution, it seems fairly safe to assume that the forces that stabilize membrane protein structure do not differ from those that stabilize water-soluble proteins in any major way and thus that membrane proteins are not structurally very different from water-soluble ones. As for the mechanisms by which membrane-bound transport enzymes catalyze their chemical reactions, high-affinity transition state binding must certainly be the central feature for these enzymes as it is for soluble enzymes, and in view of the above comments regarding the key role of interdomain movements in substrate access and transition state affinity, it is reasonable to expect that formally similar events occur during the catalysis of chemical reactions by transport enzymes. It is only a moderate extension of this line of reasoning to suggest that transporters that do not catalyze chemical reactions nevertheless undergo interdomain movements analagous to those of the arabinose-binding protein that occur upon ligand binding.

On the basis of the foregoing comments, the first postulate of the general transport paradigm is that all transporters undergo ligand-induced and -stabilized interdomain movements or cleft closures leading to sequestration of the bound ligand or ligands that induce the conformational changes away from the aqueous medium on the side of the membrane from which the ligand(s) approached. The proposed cleft closures can be thought of as hinge-bending maneuvers between two domains of a single polypeptide chain, but it is also conceivable that the same effects could be accomplished by intermonomer movements in dimers, etc., by analogy with enzymes such as glutathione reductase (165) and alcohol dehydrogenase (31), which have active sites consisting of residues from two identical monomers. However, evidence put forth in support of a requirement for dimers or higher oligomers for function in transporters is equivocal $(66,82$, $91,180)$, and as there is evidence in the case of the sarcoplasmic reticulum $\mathrm{Ca}^{2+}$-translocating ATPase, and in the case of bacteriorhodopsin, that the monomeric forms of these transporters can catalyze all of the reactions of the transport cycle $(4,25,101,180)$, in the absence of solid evidence to the contrary, it is simplest to attempt to visualize the process in terms of interdomain movements in monomers. The interdomain movements may be relatively sweeping as in the case of the arabinose-binding protein (133), or they may be much more subtle as in the case of lysozyme (62), and interdomain movements such as the interhelical movements suggested by Tanford $(179,180)$ may also be sufficient, but the important distinction to be made is that the conformational change is driven and stabilized by ligand binding, as has been most clearly shown in the case of the arabinose-binding protein.

If it is assumed that transport catalysts do undergo ligandinduced interdomain movements akin to hinge-bending cleft closure with the resultant sequestration of the inducing ligand(s) away from the aqueous medium on the side of the membrane from which the ligand(s) approached (referred to hereafter as the near side [124]), a molecularly explicit gating mechanism becomes immediately comprehensible. To be sure, cleft closure of a type like that of the arabinose-binding protein which leads to nearly total disappearance of the sugar into the center of the molecule would accomplish little in the way of transport. However, there is no fundamental reason to presume that a $360^{\circ}$ solid angle of ligand-protein interactions is needed to drive or stabilize cleft closure or both, i.e., that total engulfment of the ligand(s) is necessary, and it is also entirely possible that cleft closure could open a new aqueous pathway for diffusion in the molecule that does not exist when the protein is in the open, unliganded form. If that pathway were to extend from the binding site to the other side of the membrane (the far side), then cleft closure in itself would be a gating mechanism. Postulate two is that ligand-induced cleft closure opens an aqueous diffusion pathway from the binding site to the far side of the membrane. Stages 1 to 3 of Fig. 1 symbolize these events. When unliganded, the generalized transporter is predominantly in an open position (stage 1) ready for cleft closure. Although there may be exceptions, the general tendency to remain in the open position in the absence of the ligand or ligands that induce cleft closure more or less precludes the possibility of transporters acting as specific or nonspecific leak pathways and may contribute to their catalytic efficiency (135) as well. Upon binding of the generalized ligand (pentagon) to a part of the incomplete ligand binding site, cleft closure occurs as a result of entropic and enthalpic forces (referred to hereafter as favorable bonding interactions) comparable to those thought to drive and stabilize cleft closure of the arabinosebinding protein $(41,98,99)$. When the complete binding site with ligand in place has formed, it is no longer accessible from the near side, but is accessible from the far side, as symbolized in stage 3 of Fig. 1. It is, of course, entirely possible that the gating process could be somewhat more complex than that schematized in Fig. 1. For example, a second hinge-bending maneuver via a separate hinge region occurring concomitantly or sequentially could be needed to 


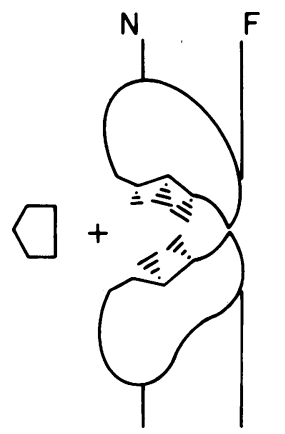

1

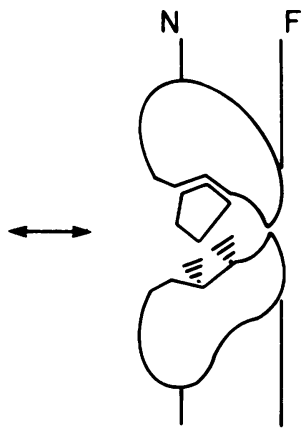

2

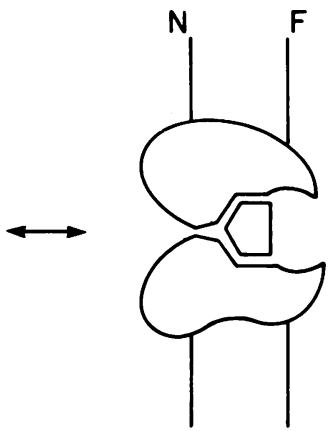

3

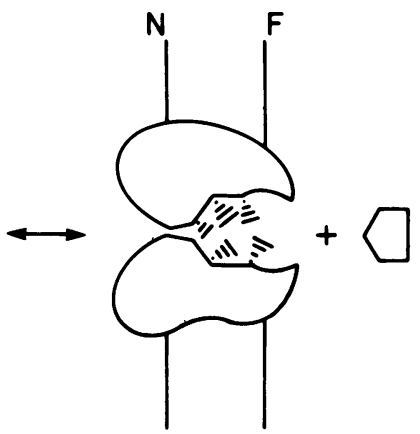

4

FIG. 1. The general transport paradigm. $\mathrm{N}$ and $\mathrm{F}$ indicate the near and far sides of the membrane, respectively. The pentagon represents the generalized ligand. The parallel lines indicate the potential for participating in favorable bonding interactions with specific atoms of the generalized ligand. See text for additional definitions and details.

open and close the aqueous diffusion pathway between the ligand-binding site and the far side, or even more complicated movements could be involved. The possible existence of such additional interdomain movements does not, however, alter the fundamental concept of gating via the hingebending maneuver; therefore, making use of Occam's razor, the simplest models with a single hinge-bending maneuver will be used throughout this article.

The events postulated thus far may collectively be termed the condensation reaction, and they occur in a thermodynamically downhill process in the presence of adequate amounts of ligand(s). The result of the condensation reaction is "translocation" of the binding site and its bound ligand(s) from access on the near side to access on the far side of the separating membrane. The third and final postulate is that, after the condensation reaction, events can occur that result in the dissipation of the favorable bonding interactions that drive and stabilize cleft closure, and in most cases, as a result of these events, which shall collectively be termed the displacement reaction, reopening of the cleft to the near side becomes the thermodynamically favored process. Using the same analogy as before, the displacement reaction can be likened to changing $L$-arabinose to $D$-fucose while it is deep within the binding site of the arabinose-binding protein. Stage 4 of Fig. 1 symbolizes one kind of displacement reaction, debinding of the transported ligand on the far side, just after it has happened and before reopening of the cleft to the near side (stage 4 to stage 1 ) has occurred. As will become obvious in the ensuing paragraphs, for each individual transport system, it is the identity of the ligands that induce the cleft closure and the precise nature of the displacement reaction that govern what is transported and what the direction of that transport is, and not always is the ligand that drives cleft closure the one that is ultimately transported. Nevertheless, the recurrent theme underlying all of the transport models to be discussed is that transport occurs via a two-stroke cyclic process consisting of a condensation reaction which originates on the near side and involves cleft closure driven by favorable bonding interactions between the transporter and one or more ligands on the near side, followed by a displacement reaction, which originates on the far side and diminishes the forces that stabilize cleft closure, and which in most cases facilitates the reopening of the cleft to the near side.

To put these notions into play, I now proceed with some explicit speculation as to how a number of different transport systems might operate within the guidelines of this general transport paradigm.

\section{SPECIFIC TRANSPORTERS}

\section{Facilitated Diffusion Systems}

Facilitated diffusion systems are defined as those transporters that require only thermal energy for their operation and are thus non-accumulative. These transporters are widespread in nature, having been identified in a variety of cells and tissues. The glucose transporter of animal erythrocytes is perhaps the most thoroughly studied example $(69,72,78$, 187). Facilitated diffusion transporters can be treated together as a class, and because the displacement reaction involves little more than thermally driven diffusion away from the binding site on the far side, the general diagram of Fig. 1 suffices as a visual aid. The condensation reaction is driven by favorable bonding interactions between the substrate to be transported (pentagon), and as a result the substrate appears in the binding site at the far side surface. As is always the case with proteins and their bound ligands, thermally driven fluctuations of the relative positions of all of the atoms of the complex are continuously taking place; accordingly, the tightness of binding fluctuates around an average value. As a result, the transported molecules are sometimes energetic enough to be released to the far side. The frequency of this thermally driven displacement reaction depends upon the strength of the ligand-transporter interactions. When the ligand is released, it, or another like it, may rebind, or, alternatively, the transporter may return to stage 1 , i.e., reopen to the near side unliganded, which it tends to do, in most cases, when the ligand is missing from the binding site. When the concentration of ligand in the aqueous phase on the far side is low, as it would be in the early part of a facilitated diffusion time course, or in the event that the ligand is metabolized on the far side, the rate of rebinding is low and a net unidirectional, near to far side flux, possibly but not necessarily, dependent upon the rate of unliganded transporter reopening, otcurs. However, as the concentration of ligand on the far side increases, as it would in the later stages of a facilitated diffusion time course of uptake of a non-metabolizable ligand, rebinding occurs more frequently, which slows the net unidirectional near-to-far side flux and enhances the far-to-near side flux via a pathway proceeding sequentially through stages $4,3,2$, and 1 of Fig. 1. Finally, when the concentrations of the ligand on the near and far sides become equal, the near-to-fat side and far-tonear side fluxes become equal. Thus, without the input of energy in some form, the transporter will only catalyze the equilibration of the ligand between the aqueous phases on the near and far sides of the membrane. This state of affairs 
will exist even though the transporter may well tend to reside predominantly in the open state (stage 1) in the absence of ligand, as a result of an intrinsic resistance to cleft closure analogous to that seen with the arabinose-binding protein. This is because, in accord with the principle of microscopic balance (196), the far side binding/debinding rate constant ratio (affinity) will be greater than the near side binding/debinding rate constant ratio by an amount equal to the ratio of the unliganded cleft opening/cleft closing rate constants. Or, in other words, if there is a tendency for the unliganded transporter to exist predominantly in the open form, and if ligand binding can overcome this tendency, then the effectiveness of far-side ligand binding to the transporter in the closed state, i.e., stage 4 , at a given concentration of ligand will be greater than the effectiveness of near-side binding of the ligand to the transporter in stage 1 , by an amount corresponding to the binding energy needed to overcome the resistance of the transporter to cleft closure. As a result of this interrelationship, at a given concentration of ligand, transporters in the conformation depicted by stage 4 may be able to bind the ligand on the far side and release it on the near side more rapidly than transporters depicted by stage 1 can catalyze the reverse process, but to the extent that they can do this, the net rate of unliganded isomerization from stage 1 to stage 4 will be proportionally less. As a consequence, when the concentrations of the ligand on the near and far sides become equal, the far-to-near and nearto-far side fluxes will be equal, even if the unliganded transporter tends to be asymmetrically distributed between the two faces of the membrane. This will be the case regardless of the relative magnitudes of the various rate constants, although, as will be mentioned again below, certain combinations of rate constants could make the approach to equilibrium extremely slow. However, it is important to note that the values obtained for unidirectional flux rates from one side at a given concentration of ligand to the other side containing no ligand (i.e., a so-called zero trans experiment) do depend upon the relative magnitudes of these rate contants. A steady-state analysis of the model shown in Fig. 1 by the King-Altman method (81), as explained by Hill (58), shows clearly that the zero trans near-to-far side flux rates may be either significantly greater than or less than the far-to-near side flux rates at a given concentration of ligand, depending upon the values of the individual rate constants. Such behavior may well be at the root of kinetic curiosities such as the "K paradox" discussed by Wilbrandt (192) and LeFevre (93) and the often observed phenomenon of accelerative exchange diffusion (171).

As an extension of this particular line of reasoning, it is pertinent to point out that it is possible that a transporter may be of such a nature that reopening to the near side in the absence of ligand (stage 4 to stage 1) occurs only with great infrequency. Such a transporter would be useless as a catalyst of predominantly one-way ligand movements as would be needed, for example, in the case of nutrient transport systems, because it could only catalyze net transport at a rate equal to the rate at which it opened to the near side in the absence of ligand. However, such a transporter could readily catalyze the countermovement of another ligand with adequate affinity, or the same ligand, if either were already present in sufficient quantity on the far side. A transporter of this type would account for the kind of behavior seen with the obligate anion-exchange transporter in the erythrocyte plasma membrane $(66,96,153-155)$. The adenosine diphosphate (ADP)/ATP exchanger in the mitochondrial inner membrane (82-84) may also be of this type.
As one final note along these lines, it should be mentioned that, although a similarity between the arabinose-binding protein and membrane-bound transporters has been implied thus far, there must be significant differences as well. Obvious differences, already inferred, are that transporters must have a hydrophobic region on their surface to anchor them in the membrane and that they must be designed such that their binding site accessibility from the near and far sides of the membrane can change. Moreover, at least for facilitated diffusion transporters, there must be an additional fundamental dissimilarity. The energy difference between the closed, liganded and closed, unliganded forms of the arabinose-binding protein is about $50 \mathrm{kcal} / \mathrm{mol}$, and it is obvious that a facilitated diffusion transporter with analogous properties would face insurmountable kinetic problems, because with such an energy difference the probability of debinding the ligand on the far side would be vanishingly low (34), even if a free path for diffusion away from the binding site existed. Presumably, therefore, if facilitated diffusion transporters work as postulated here, they would have evolved to operate with significantly smaller differences between the probability of existence of the closed, liganded and closed, unliganded forms, i.e., stages 3 and 4 of Fig. 1, respectively. This would appear relatively straightforward to do as the primary reasons for the vast extremes in the case of the arabinose-binding protein can be traced to only a few charged residues (98). The solvation energy of these residues is thought to stabilize the open, unliganded form relative to the closed, unliganded form; in addition, the entropic gain from the release of several water molecules upon bonding of three of these residues with atoms of the arabinose molecule stabilizes the closed, liganded form with respect to the open, unliganded form. These interactions acting in concert account primarily for the large energy difference between the closed, liganded and closed, unliganded forms. An analogous protein with fewer of such charged groups would be expected to have a substantially smaller energy difference between the closed, liganded and the closed, unliganded forms and would, accordingly, be more suitable as a facilitated diffusion transporter because of its greater net tendency to release the ligand when facing the far side. On the other hand, accumulative transporters may not be as subject to such constraints because, as outlined in detail below, debinding on the far side is assisted by the energy input or because most or all of the ligands that drive cleft closure do not debind on the far side, or for both reasons.

\section{Transporters Coupled to Electrochemical Ion Gradients}

This class of transporter comprises the numerous transporters that catalyze the transmembrane accumulation of solutes at the expense of transmembrane chemical or electrical ionic potential differences (or both), most commonly those of $\mathrm{H}^{+}$or $\mathrm{Na}^{+}$, that are generated by physically separate (presumably) proton or sodium pumps. When the accumulation takes place on the side of the membrane with the relatively low electrochemical potential of the driving ion, the process is known as symport (113). Wellcharacterized symporters include the $\mathrm{H}^{+}$-galactoside symporter in the plasma membrane of $E$. coli $(74,138)$ and the $\mathrm{Na}^{+}$-glucose symporter in the plasma membrane of mammalian intestinal epithelial cells $(20,59,80,166)$, to name just two. When the accumulation occurs on the side with the relatively high electrochemical potential of the 
driving ion, the process is known as antiport (113). Several established members of this group are the $\mathrm{Na}^{+} / \mathrm{Ca}^{2+}$ antiporters of many animal cells $(8,9,148)$, the $\mathrm{H}^{+} / \mathrm{Ca}^{2+}$ antiporters of fungal $(176,177)$ and bacterial $(7,11,152)$ plasma membranes, the $\mathrm{Na}^{+} / \mathrm{H}^{+}$antiporters of bacteria (89), and the $\mathrm{H}^{+}$/catecholamine antiporter of mammalian cell catecholamine storage vesicles $(44,67,68,76,163)$.

Figure $2 \mathrm{~A}$ outlines the key stages of the generic symport reaction; the driven ligand (lactose, glucose, etc.) is represented by the pentagon, and the driving ion is symbolized as $\mathrm{C}^{+}$. As with all of the other models of Fig. 2, only the active site is shown. The postulated scheme is very similar to that discussed above for the facilitated diffusion systems with the single additional feature that occupancy of the driving ionbinding site by the driving ion facilitates the binding of the driven ligand and subsequent cleft closure. The symporter condensation reaction is symbolized by the first two stages in Fig. 2A. After the cleft has closed, the driven ligand and the driving ion both may debind on the far side, and as was the case with the facilitated diffusion systems, the frequency of debinding depends upon the strength of the interactions between the driven ligand, the driving ion, and the transporter. Such debinding constitutes the symporter displacement reaction, the result of which is shown in stage 3 of Fig. 2A. Finally, after the displacement reaction, the transporter is inclined to reopen unliganded to the near side as depicted in stage 4. Providing that the rate constants for all of the steps in the cycle are of sufficient magnitude, in the absence of a transmembrane electrochemical potential difference of the driving ion, the transporter will catalyze the coupled facilitated diffusion of the driven ligand and the driving ion. However, in the presence of such a potential difference, near side positive and more concentrated, for example, the result will be different. The relatively high electrochemical potential of the driving ion on the near side will favor binding of the driving ion, and hence binding of the driving ligand, on the near side, and, conversely, the relatively low electrochemical potential of the driving ion on the far side will favor its debinding, and hence that of the driven ligand, on the far side. In addition, if present, a phase-specific electrical potential difference may also influence the process by facilitating the near-side binding and far-side debinding of the driven ligand, it is appropriately charged, and by facilitating either the reopening of the unliganded transporter to the near side or its liganded closing to the far side, or both, depending upon the net charges of the liganded and unliganded forms of the transporter. For example, if the driven ligand is uncharged, the driving cation is monovalent, and the ternary complex bears a single net positive charge, as implied in Fig. $2 \mathrm{~A}$, then cleft closure will be promoted by a near-sidepositive electrical potential difference, but cleft reopening will be indifferent to the electrical potential because the unliganded transporter (stage 3 ) will be electrically neutral. Alternatively, if the ternary complex is electroneutral, then cleft closure will not be aided by a near-side-positive electrical potential difference, but cleft reopening will be, because the unliganded transporter will bear a single net negative charge. Obviously, many other permutations are conceivable depending upon the charges of the driven ligand, the driving ion, and the binding site residues, and the stoichiometry of the reaction. It is also clear from such considerations that the energy input may affect binding site availability as well as binding site affinity (138). And, it should also be added that all of the effects of the transmembrane electrochemical potential difference of the driving ion may well be exerted solely via residues in or near the ligand-binding (active) site, because, at least in the simplest formulations, these are the only residues that undergo the change in accessibility from the aqueous media on the near and far sides of the separating membrane that is required for coupling to occur. This general question of the directness of coupling between the driving and driven particles will be addressed in somewhat more detail below. In any case, the combined result of most or all of the abovementioned effects of the energy input is sym coupling of the flows of the driving ion and the driven ligand and accumulation of the driven ligand on the far side at a higher electrochemical potential. If the driven ligand is uncharged, this of course simply means accumulation against a concentration gradient. The thermodynamic coupling relationships for such systems have been thoroughly and elegantly developed and described by Mitchell $(115,119)$.

For reasons similar to those mentioned above for facilitated diffusion systems, symporter flux kinetics are expected to be unpredictable and of uncertain meaning in the absence of complete knowledge of the values of all of the individual rate constants, in both the presence and the absence of a transmembrane electrochemical potential difference of the driving ion. Moreover, by virtue of the fact that symporters catalyze the flow of more than one species, additional kinetic complications may arise as a result of the catalysis of partial reactions such as ligand-induced cleft closure and debinding of the ligand on the far side without participation of the driving ion, or cleft closure as shown in Fig. 2A followed by debinding of only the driving ion or the driven ligand on the far side and reopening to the near side with the other particle still bound, or both. Similar potential kinetic complications exist with all of the remaining transporters to be discussed in this article, and having acknowledged this fact, little further specific reference to kinetic phenomena will be made. It might be mentioned in this context, however, that once the possibility of partial reactions such as those mentioned above is recognized, the often observed conditiondependent or noninteger values of transport stoichiometries $(30,86)$ become particularly straightforward to rationalize. And, as one final commentary related to this general drift, it is to be noted that, as was the case with the facilitated diffusion systems, depending upon the relative values of each of the individual rate constants, transporters of the general type schematized in Fig. 2A can have very different properties and serve very different cellular functions. For example, a symporter that can readily debind the driven ligand on the far side upon debinding the driving ion and can freely reopen in the unliganded form to the near side will be an efficient catalyst of nutrient accumulation in a cell that can generate a transmembrane electrochemical potential difference of the driving ion of the proper polarity. On the other hand, a symporter that can readily debind the driving ion on the far side but cannot readily debind the ligand, and thus reopens to the near side with the ligand bound, would be useless for nutrient accumulation and might well be detrimental to a cell because it would act as a liganddependent dissipator of the ion gradient. Such a transporter could, however, serve a vital role in cell-to-cell communication in its capacity to act as a ligand-dependent ion channel. The nicotinic acetylcholine receptor $(15,19)$ may be an example of such a transporter. The frequency at which hinge-bending conformational changes can take place $(77$, 110) appears to be more than adequate to account for the ion flux kinetics observed for even the fastest ion channels. It only remains to mention that little need be added to the argument to imagine similar mechanisms for ion channels 


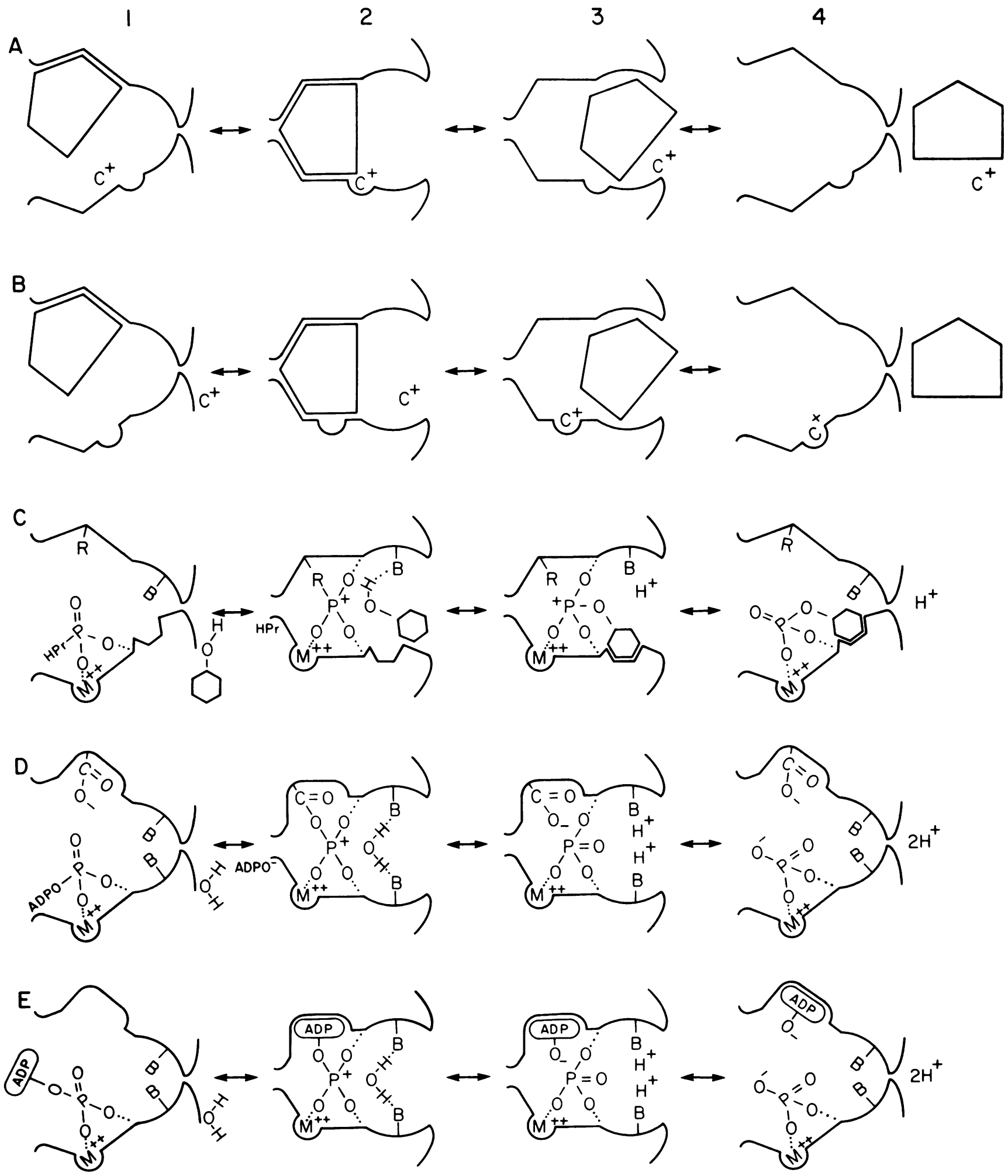

FIG. 2. Specific transporter mechanisms. Only the active sites of each transporter are shown in the diagrams. The left-hand side of each active site is the near side and the right-hand side of each is the far side. Favorable bonding interactions such as hydrogen bonds, purely electrostatic attractions, hydrophobic forces, entropic gains, etc., are indicated by dotted lines, complementary fits, or occupancy of pockets in the various active sites. $\mathrm{M}^{2+}$ indicates a divalent cation. In $(\mathrm{G})$, the proximity of $\mathrm{K}^{+}$to neighboring atoms implies attractive interactions. Covalent bonds are indicated as solid lines. Stages 1 to 4 are not precisely the same as in Fig. 1. See text for additional definitions and details. 

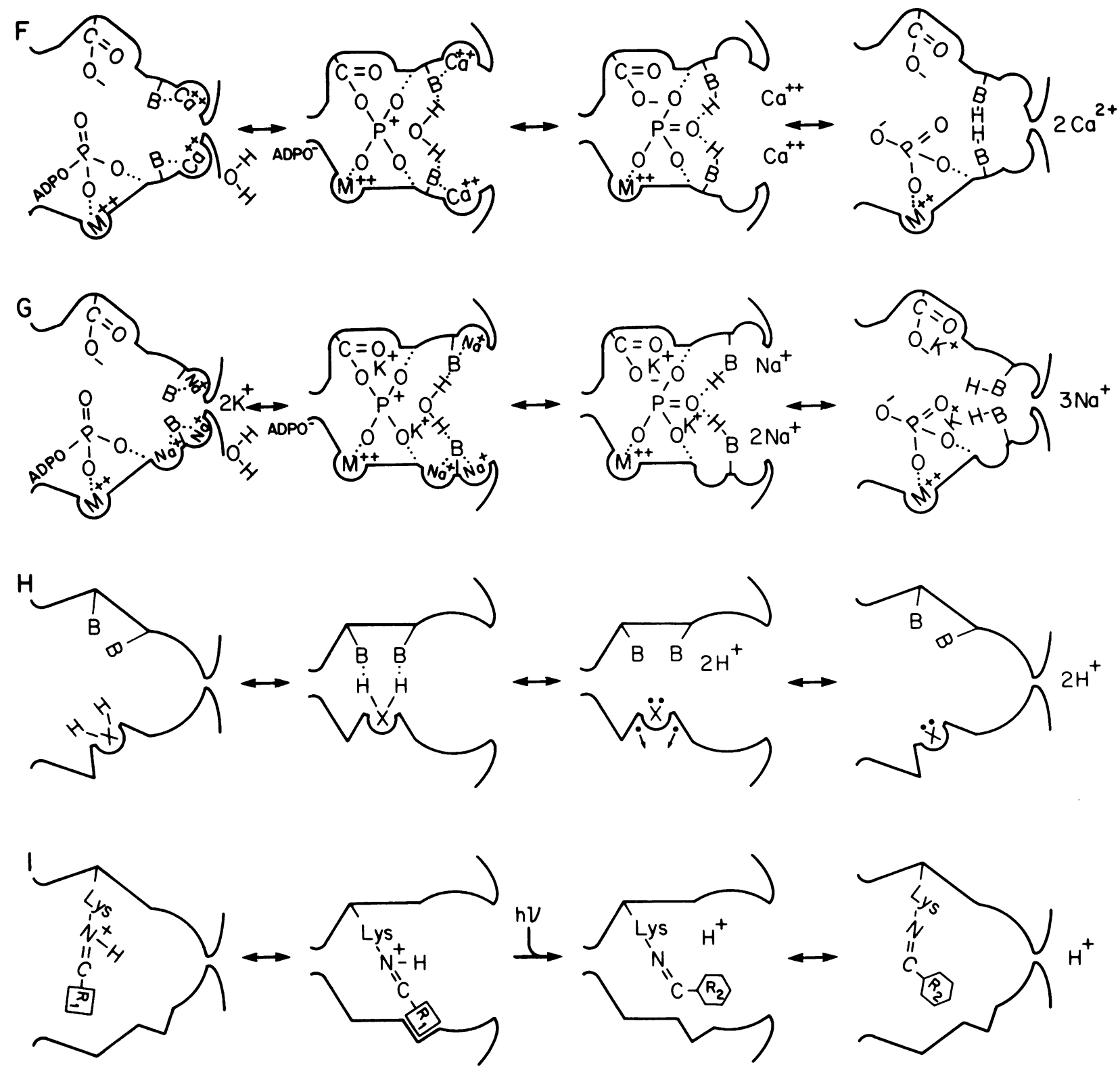

responsive to signals other than chemical ligands. The possible similarity between transporters and channels has been elaborated upon by Läuger (92).

Figure 2B shows the generic antiport reaction. Here, it is the binding of the ligand alone that drives the condensation reaction, and it is further stipulated that the ligand binding and cleft closure take place more efficiently when the binding site for the driving ion is empty than when it is occupied by the driving ion. Thus, given adequate values for the various rate constants, facilitated diffusion of the driven ligand and the driving ion can occur, and when the antiporter operates in the accumulative mode, i.e., in the presence of a transmembrane electrochemical potential difference of the driving ion, near side negative and less concentrated, for example, unloading of the binding site for the driving ion and hence binding of the driven ligand are favored by the relatively low electrochemical potential of the driving ion on the near side; conversely, the relatively high electrochemical

potential of the driving ion on the far side favors binding of the driving ion and hence displacement of the ligand on the far side, which increases the tendency of the transporter to reopen to the near side with the driving ion in place. In addition, as with the symporter, the electrical potential difference may facilitate binding and debinding of the driven ligand if it is appropriately charged, and the opening or closing of the cleft or both. The net result here is anti coupling of the flows of the driving ion and the driven ligand with the equilibration of the ligand on the far side at a higher electrochemical potential, to an extent dependent upon the stoichiometry of the reaction and the magnitude of the electrochemical potential difference of the driving ion.

\section{Transporters Coupled to Chemical Transformations}

The third major class of transporters comprises those that catalyze the accumulation of various ions and other ligands 
against electrochemical gradients utilizing the energy made available from certain chemical reactions. Major members of this class are the ion-translocating ATPases such as the $\mathrm{F}_{1} \mathrm{~F}_{O}$ $\mathrm{H}^{+}$-ATPase/ATP synthases of mitochondria, bacteria, and chloroplasts $(2,38,43,75,88,97,137,167)$, the $\mathrm{Na}^{+} / \mathrm{K}^{+}$. translocating ATPase of animal cell plasma membranes (48, 70), the $\mathrm{Ca}^{2+}$-translocating ATPase of sarcoplasmic reticulum $(24,178,181)$, the $\mathrm{Ca}^{2+}$-ATPases of eucaryotic and procaryotic plasma membranes $(13,14,104,160)$, the $\mathrm{H}^{+} / \mathrm{K}^{+}$-translocating ATPase of gastric mucosa $(35,156)$, the $\mathrm{H}^{+}$-translocating ATPases in the plasma membranes of fungi and plants $(49,146,159,168)$, the $\mathrm{K}^{+}$-ATPase of $E$. coli $(33,57)$, and the organellar $\mathrm{H}^{+}$-ATPases $(5,39,68,136,175)$. Other major members of the chemical transformation class of transporters are the bacterial phosphoenolpyruvatedependent sugar phosphotransferase systems $(151,157)$, the redox proton pumps in respiratory chains and elsewhere (18, $37,50,126,191)$, and the light-driven ion pumps (162, 172-174). Momentarily, I shall proceed to a detailed consideration of how these systems may operate in conformity with the general transport paradigm described earlier, but at this particular point in the discussion, it is appropriate to digress briefly and reflect upon the general question of coupling between the flows of substances that drive accumulative transport and those that are accumulated.

All transporters catalyze flows of particles, be they ions, molecules, electrons, photons, or chemical groups, between two or more states. The transporters discussed thus far catalyze either single or coupled flows of particles between the aqueous phases on either side of a separating membrane. The transporters to be discussed in the ensuing paragraphs catalyze similar flows between the aqueous phases on either side of a separating membrane, and flows of particles between chemical states (chemical and photochemical reactions) as well. For all transport reactions that involve the coupled flow of more than one type of particle, it is a central mechanistic question as to how the flows are coupled, as they must be if the flow of one particle from a higher to a lower potential is to be used to drive another particle from a lower to a higher potential. Opinions as to how the particle flows might be coupled are best dichotomized by reference to the two possible extreme cases, which shall be called direct and completely indirect coupling, using Mitchell's terminology (121). In the case of completely indirect coupling, the effect of the driving reaction or particle flow is conceived as being exerted entirely "at a distance" via conformational changes in the transporter molecule. Expositions of this point of view are predominant in the history of transport; the most recent advocate of such conformational coupling is Tanford $(179,180)$. In the alternative case of direct coupling, the flow of particles that constitutes the driving reaction is contiguous with the flow of the driven ligand, on a scale of dimensions much smaller than the dimensions of the entire transporter molecule. That is, both flows occur through virtually the same active site. The primary protagonist of direct coupling is Mitchell (e.g., 112, $114,116-118,120,121,123-125,127-129)$; models originating from this laboratory $(22,158,159)$ also support this view. It would appear, at least on the surface, that either viewpoint is of roughly equal potential as a representative of the real situation. Tanford has opted for indirect coupling on the grounds that it is less complex, that there is evidence in support of it, and that there is no evidence in support of direct coupling (180). However, it must be noted that the validity of each of these notions is debatable. First, simply stipulating that a protein accomplishes coupling indirectly via conformational changes without specifying an explicit biochemical mechanism does not make the indirect mechanism less complex. And, in fact, when attempts are made to formulate explicit indirect coupling models, it is quickly seen that they must be more complex than their direct counterparts, because they must describe not only all of the atomic movements involved in the driving and driven reactions, but all of those involved in propagating the action at a distance as well. Second, although there is ample evidence for the occurrence of conformational changes in transporter molecules as they proceed through their various transport cycles, the fact that such conformational changes occur is not evidence that they indirectly couple the various particle movements, for similar conformational changes are expected in direct coupling mechanisms, as is seen repeatedly in this article. Indeed, until complete angstrom-level structural information for a transporter molecule at several stages of its catalytic cycle becomes available, it is difficult to even imagine how unequivocal evidence for indirect coupling could ever be obtained, at least for most transporters. Thus, since no such information is yet available, it is unlikely that any solid evidence in favor of indirect coupling is extant. Finally, it can be pointed out that, whereas there is no evidence for indirect coupling, there is evidence available in support of direct coupling. In the case of the $\mathrm{Na}^{+} / \mathrm{K}^{+}$translocating ATPase, there is evidence for angstrom-level propinquity between the active site and at least some of the transported ions (51-53). Even more conclusively, in the case of the bacterial phosphoenolpyruvate-dependent sugar phosphotransferase systems, we know that the transported sugars are intimately associated with the enzyme active site, because they are phosphorylated during their transport (73). It can therefore be argued that if transport is to ultimately be understood as a process with a fundamentally universal mechanism, then efforts to explain transport coupling by direct mechanisms should be more lucrative than efforts in the opposite direction. With this in mind, we can now proceed to speculation as to how the chemical transformation-type transporters might work, and, in view of the foregoing comments, it is appropriate to begin with the bacterial phosphoenolpyruvate-dependent sugar phosphotransferase systems.

Figure $2 \mathrm{C}$ presents a model for the transport reaction catalyzed by the various phosphotransferases formulated within the guidelines of the general transport paradigm presented earlier. The model is also formulated to take into account certain more or less established biochemical features of these systems, including the stoichiometry of the reaction, the covalent phosphoryl-enzyme intermediate $(6$, $61,111,145)$, and the divalent cation requirement (111). This has been done for each of the remaining models of Fig. 2, although specific mention of all of these properties will not always be made. In reactions not shown, the phosphorylated form of the small protein $\mathrm{HPr}$ has been generated from phosphoenolpyruvate in a reaction catalyzed by the enzyme known as enzyme I. The sugar-specific transporter, known as enzyme II, then binds the phosphoryl-HPr, or an additional intermediate, phosphoryl-enzyme III, that has been phosphorylated by phosphoryl-HPr $(151,157)$ and undergoes cleft closure driven by favorable bonding interactions between the phosphorylium group (95) donor and enzyme II. During this condensation reaction (stages 1 and 2 ), the phosphorylium group is transferred to an acceptor group, designated by the $R$, in the active site of the enzyme II via a transition state in which the enzyme-ligand interactions are at maximum strength $(65,94,144,194,195)$. Although the 
liberated HPr is shown as leaving to the near side just after the first transphosphorylation reaction, it may also remain associated until a later stage of the catalytic cycle. As with the other transporters that have been discussed thus far, the condensation reaction results in the opening of an aqueous pathway between the active site and the far side, in preparation for the displacement reaction. However, in this case, the ligand that drives the cleft closure (the phosphorylium group predominantly) is not destined to be displaced on the far side, but rather is positioned to accept the sugar moiety (hexagon-OH) after it diffuses from the far side into the sugar-binding site designated by the hexagonal depression and the general base, $B$. In stage 2 , the initial contact between the sugar and its binding site has occurred, and in stage 3 the covalent bond rearrangement has taken place, via a second transition state, with the formation of enzymebound sugar phosphate and liberation of the sugar proton assisted by the general base, B. For both the enzyme and sugar phosphorylation reactions, the central features of the phosphoryl transfer catalysis including electron withdrawal by electrophilic centers and proton withdrawal by the general base, both of which are signified by the dotted lines, are modeled after the general phosphorylium group transfer mechanism of Knowles (85). Upon the formation of the phosphorylated sugar, the displacement reaction is complete, and as was the case with the other transport systems discussed thus far, as a result of the displacement reaction, some of the favorable bonding interactions that drove cleft closure no longer exist. For this reason, reopening of the cleft is favored (stage 4), after which debinding of the phosphorylated sugar on the near side can occur. As a side issue, the fate of the sugar proton is worthy of some attention. It is entirely possible that the general base $B$ is of a chemical nature or in a position in the active site such that release of its bound proton to the aqueous medium on the far side is highly unlikely. If so, the proton would be expected to return with the rest of the binding site to the near side as a result of cleft opening and be released with the phosphorylated sugar more or less unnoticed. However, it is equally possible that the general base $B$ could be of a chemical nature or in a position in the active site such that release of its bound proton to the aqueous medium on the far side does occur. If this were the case, the transporter would be an electrogenic proton pump in addition to a vectorial sugar phosphorylation system. The transport reaction is formulated in this way in Fig. $2 \mathrm{C}$ to raise this possibility as an explanation of the experimentally observed inhibition of phosphoenolpyruvate-dependent sugar phosphotransferase systems by a transmembrane electrochemical protonic potential difference and the release of this inhibition by proton conductors (149).

From the postulated sugar phosphotransferase mechanism described in Fig. 2C, a model for the mechanism of the ATP hydrolysis-coupled electrogenic proton pumps of the aspartyl-phosphoryl-enzyme intermediate family $(49,146,159$, 168 ) is readily evolved, with little that is conceptually different. Figure 2D shows such a formulation. Here, instead of phosphorylated HPr, ATP (or Mg ATP) is the compound possessing the structural and chemical attributes that the transporter requires for cleft closure and formation of the phosphoryl-enzyme intermediate (the condensation reaction) to occur. ADP is released on the near side sometime during the cycle as was $\mathrm{HPr}$ in Fig. $2 \mathrm{C}$, and as with all the other condensation reactions, the result is the establishment of a pathway between the active site and the medium on the far side (stage 2). At this point, the hydrolytic water mole- cule is shown as having moved into position for the aspartylphosphoryl-enzyme intermediate hydrolysis reaction, which is assisted by proton withdrawal by the two general bases, designated again by the letter B. In stage 3, the aspartylphosphoryl-enzyme intermediate hydrolysis reaction has been completed. This reaction is strictly analagous to the one above it, except that the oxide ion of water is transferred to the phosphorylium group rather than the hexose anion, and both water protons have been removed for release to the far side. Thus, as drawn, the stoichiometry of the transport reaction would be two protons appearing on the far side per ATP hydrolyzed. If, or perhaps when is a more appropriate term (185), this stoichiometry is one $\mathrm{H}^{+}$per ATP hydrolyzed, then the alternative formulation would involve the transfer of a water hydroxyl ion to the phosphorylium group and proton withdrawal by only one $\mathrm{B}$. In either case, as before, after the displacement reaction is over, the forces tending to hold the cleft closed are diminished, and the cleft reopens, with subsequent release of the other product of the reaction, inorganic phosphate, to the near side. Finally, it should be noted that, as drawn, the water protons are released to the aqueous medium on the far side from B's that become exposed to the aqueous medium on the near side after the cleft reopens. In an alternative formulation presented previously (159), the B's were visualized as existing in a part of the transporter polypeptide chain that is in the active site when the cleft is closed but makes contact with the far side when the cleft is open. Either of these possibilities seems reasonable in the absence of experimental support for or against one or the other. And, on that note, it is a particularly opportune point in the discussion to remind the reader that all of the models shown in Fig. 2 are presented with the notion that the provision of some detail will serve to better illustrate the basic principles and relative simplicity of direct coupling mechanisms and to show how virtually all transport mechanisms can be viewed within the same conceptual framework. It is by no means imagined that the detailed atomic positions and movements presented in the diagrams could be anything more than rough approximations of reality.

A direct coupling mechanism for the $\mathrm{F}_{1} \mathrm{~F}_{O} \mathrm{H}^{+}$-ATPase/ ATP synthases of mitochondria, bacteria, and chloroplasts formulated within the guidelines of the general transport paradigm is presented in Fig. 2E. These enzymes differ from the proton pumps of the aspartyl-phosphoryl-enzyme intermediate family of transport ATPases in that they do not have a covalent phosphoryl-enzyme intermediate (186), and they are also structurally more complex, with multiple cooperatively interacting hydrolytic subunits and a separate protonconducting channel. These features no doubt contribute to the ability of these enzymes to efficiently perform their dual physiological roles of ATP-dependent proton translocation and ATP synthesis in response to a transmembrane electrochemical proton gradient in a rapid and regulated manner; nevertheless, the molecular events occurring at the individual active sites of these enzymes (possibly in an interdomain cleft in each $\beta$ subunit [79]) may be fundamentally quite similar to those of the aspartyl-phosphoryl-enzyme intermediate family, as the diagram intends to show. Since there is no covalent intermediate, the condensation reaction for these pumps involves only ATP (or Mg ATP) binding as depicted in stages 1 and 2. The displacement reaction, cleft reopening, and product release then follow in a manner analogous to that described above for Fig. 2D except that the protons are released into the $\mathrm{F}_{o}$ proton channel. For more or less arbitrary reasons, the stoichiometry of the reaction 
shown in Fig. 2E is $2 \mathrm{H}^{+}$appearing on the far side per molecule of ATP hydrolyzed. If the stoichiometry is actually $3 \mathrm{H}^{+}$translocated per ATP hydrolyzed instead of 2 , it would be necessary to stipulate the participation of an additional proton in the favorable bonding interactions that drive cleft closure and the dislodging of that proton on the far side as a result of the displacement reaction. Alternatively, the farside to near-side movement of an additional hydroxyl ion as a result of its participation in the displacement reaction is also plausible. A possible site for such hydroxyl ion involvement is the enzyme-bound divalent cation. And just to iterate an important general point made earlier, if the overall sequence of reactions is not entirely obligatorily coupled, as, for example, would be the case if the additional proton or hydroxyl ion does not participate in every cycle, the stoichiometry may be condition dependent or have a noninteger value or both.

The operation of the $\mathrm{F}_{1} \mathrm{~F}_{o} \mathrm{H}^{+}$-ATPase/ATP synthases in the direction of ATP synthesis can also be readily visualized with the aid of Fig. 2E. After the unliganded enzyme has bound the substrates, ADP and $P_{i}$, on the near side (stage 4), it undergoes cleft closure, which opens a pathway between the active site and the base of the $F_{o}$ proton well (stage 3). At this point, a covalent bond rearrangement involving protons from the far side, the bound $\mathrm{P}_{\mathrm{i}}$, and the bound ADP occurs, leading to the formation of bound ATP and water (stage 2). The enzyme then reopens to the near side (stage 1), after which release of the bound ATP becomes possible. Presumably, as was the case above for the symporters and antiporters operating in their accumulative modes, the input energy in the form of a transmembrane proton flow driven by a transmembrane protonic potential difference, far side positive and acidic, may facilitate the process in several different ways. Thus, the relatively high protonic potential on the far side may enhance transition state binding affinity by promoting the protonation of residues involved in the extraction of the phosphoryl oxygen as shown (Fig. 2E), and, if the stoichiometry of the reaction is $3 \mathrm{H}^{+}$per ATP, the protonic pressure may also enhance the transition state affinity by assisting in the protonation of, or hydroxyl extraction from, the additional site involved in ATP binding mentioned above. Furthermore, the relatively low protonic potential on the near side may promote deprotonation or hydroxylation of the additional site involved in ATP binding, thus favoring ATP debinding and binding of ADP and $\mathrm{P}_{\mathrm{i}}$. And, as was the case for the symporters and antiporters, the phase-specific electrical potential difference may also facilitate ligand (ADP and $\mathrm{P}_{\mathrm{i}}$ ) binding and cleft closure and may in turn facilitate cleft reopening and ATP release after the bound ATP has been formed. The combined effect of many or all of these forces, exerted solely on the participants of the chemical reaction and enzyme active site residues, is a facile turning of the cycle in the direction of ATP synthesis at the expense of the transmembrane protonic potential difference. Finally, as a related issue, it is important to note that under certain experimental conditions, in the absence of a transmembrane protonic potential difference of sufficient magnitude, the individual rate constants of the process described in Fig. 2E may well be such that the cycle tends to stall at some stage, for example, at stage 2 . If so, depending upon the conditions, this could be experimentally manifest as high-affinity ATP binding and diminution of such binding upon energization of the membrane, uncoupler-insensitive exchange reactions, or unenergized synthesis of bound ATP from bound ADP and medium $P_{i}$. The fact that such reactions can readily be accounted for by a direct coupling mechanism such as that schematized in Fig. $2 \mathrm{E}$ is offered as evidence that the demonstration of energy-dependent binding affinity changes, uncoupler-insensitive exchange reactions, and unenergized synthesis of enzyme-bound ATP cannot be taken as evidence that the energy input in these enzymes is indirectly coupled to ATP synthesis, as has often been suggested (10, 21, 36). Similar points can be made for every other transporter discussed in this article. The tendency of a transporter to accumulate at a particular stage of its catalytic cycle is simply a manifestation of the values of all of the individual rate constants of the process under a given set of experimental conditions and is not diagnostic of a particular mechanistic point of view.

A model for the mechanism of the $\mathrm{Ca}^{2+}$-translocating ATPase of sarcoplasmic reticulum is readily adapted from the postulated proton pump mechanisms of Fig. 2D and E, again with only minor alterations. Such a model is shown in Fig. 2F. The condensation reaction is the same as that visualized for the proton translocating ATPases of the aspartyl-phosphoryl-enzyme intermediate family (Fig. 2D) except that the occupancy by $\mathrm{Ca}^{2+}$ of two $\mathrm{Ca}^{2+}$-binding sites is required for efficient cleft closure and the transphosphorylation reaction from ATP to the aspartate carboxyl group to occur. After the condensation reaction (stages 1 and 2), the pathway to the aqueous medium on the far side is open and the hydrolytic water molecule moves into position in preparation for the aspartyl-phosphoryl-enzyme intermediate hydrolysis reaction, which will constitute the displacement reaction. For the sake of comparison, if this were a standard indirect coupling model, the next event to occur, if it had not already, would be a conformational isomerization reaction, driven by a force of unspecified origin, that converts the calcium-binding sites from a high-affinity form to a lowaffinity form, whereupon the $2 \mathrm{Ca}^{2+}$ would be released to the medium on the far side. In contrast, in formulating a possible direct coupling mechanism for a transporter of this type, it is sought to identify one or more aspects of the chemical reaction, or as above for the symporters and antiporters, the transmembrane electrochemical potential difference and flow of the driving ion particles, that can bring about the binding site affinity or availability change or both. Without such an event, little in the way of transport, other than $\mathrm{Ca}^{2+}$ exchange between the aqueous medium on the far side and the high-affinity sites, and possibly some facilitated diffusion of $\mathrm{Ca}^{2+}$, can occur. The obvious candidate for the chemical event in this case is the aspartyl-phosphoryl-enzyme intermediate hydrolysis reaction, which is shown in the process of beginning in the second diagram of Fig. 2F. As in the two diagrams above it, the nucleophilic attack by the water oxygen atom on the phosphorous atom, which is electrophilic by virtue of electron withdrawal by several electrophilic centers in the active site (85), is facilitated by proton withdrawal by general bases designated by the B's. However, in this case, it is further stipulated that the general bases are literally part of the high-affinity calcium-binding sites. Thus, when the chemical bond exchange that constitutes the aspartyl-phosphoryl-enzyme intermediate hydrolysis reaction is completed (stage 3 ), the B's have been charged with the hydrolytic water protons, and as a result, the affinity of each of the sites for $\mathrm{Ca}^{2+}$ is decreased, which favors debinding of the $2 \mathrm{Ca}^{2+}$ and release to the aqueous medium on the far side. Then, as with all the other models, when the displacement reaction has concluded the sum of the forces tending to hold the cleft closed is reduced, which leads to cleft opening and release of the final products of the reaction, in this case inorganic phosphate and the two water 
protons, to the aqueous medium on the near side, whereupon a new transport cycle can ensue. It might be noted that there is a difference between this model and one previously proposed for the sarcoplasmic reticulum $\mathrm{Ca}^{2+}$-translocating ATPase (159), in that in the earlier formulation the B's were not exactly a part of the high-affinity $\mathrm{Ca}^{2+}$-binding sites, but rather transferred their bound protons to the high-affinity sites at some time after the aspartyl-phosphoryl-enzyme intermediate hydrolysis reaction had occurred. Furthermore, as an alternative to either of these possibilities, stipulation that the aspartyl-phosphoryl-enzyme intermediate hydrolysis reaction occurs more readily when the $\mathrm{Ca}^{2+}$. binding sites exposed to the far side are empty, allows the formulation of a model in which reduced $\mathrm{Ca}^{2+}$-binding site availability and not reduced affinity can account for the observed accumulation of $\mathrm{Ca}^{2+}$ on the far side (see reference 180 , p. 392 , for some discussion of this issue). Certain combinations of these alternatives are possible as well. This serves to illustrate again that there is a reasonable degree of flexibility as to the possible ways in which direct coupling might proceed at the molecular and submolecular levels and once more educes the important point that any proposed model of this sort must, by virtue of the obvious great uncertainty as to the exact composition of the active sites in question, be recognized as a collection of only moderately informed guesses. They are therefore, at best, only an approximation of reality, but are nevertheless of utility in that the specific postulation of chemical and molecular details may be of heuristic value to an investigator seeking to understand the meaning of a particular set of experimental observations.

The next transporter to be considered is the long-studied $\mathrm{Na}^{+} / \mathrm{K}^{+}$-translocating ATPase of animal cell plasma membranes. As for all other transporter mechanisms that have been considered in Fig. 2, the direct coupling formulation for the $\mathrm{Na}^{+} / \mathrm{K}^{+}$-translocating ATPase is similar to the one above it with only minor alterations. Thus, as diagrammed in Fig. $2 \mathrm{G}$, three $\mathrm{Na}^{+}$-binding sites have been introduced in place of the two $\mathrm{Ca}^{2+}$-binding sites of the sarcoplasmic reticulum ATPase, but the condensation reaction is otherwise identical. Similarly, the general bases that assist the aspartylphosphoryl-enzyme intermediate hydrolysis reaction are part of the $\mathrm{Na}^{+}$-binding sites, and the protonation of the general bases that results in this reaction causes a decrease in the affinity of the $\mathrm{Na}^{+}$-binding sites for $\mathrm{Na}^{+}$, which favors the release of $\mathrm{Na}^{+}$on the far side. As for the $\mathrm{Ca}^{2+}$-ATPase, an exchange of $\mathrm{Na}^{+}$between the high-affinity $\mathrm{Na}^{+}$sites and the aqueous medium on the far side, and possibly some facilitated diffusion of $\mathrm{Na}^{+}$, can occur, but accumulative transport cannot take place without the occurrence of the aspartyl-phosphoryl-enzyme intermediate hydrolysis reaction and the attendant decrease in the affinity or availability or both of the $\mathrm{Na}^{+}$-binding sites on the far side. As one final modification, the role of $\mathrm{K}^{+}$from the far side in assisting the progress of the aspartyl-phosphoryl-enzyme intermediate hydrolysis reaction has been added. Although the $\mathrm{K}^{+}$ions are shown as interacting more or less directly with the three functional groups undergoing the covalent bond exchange (i.e., the aspartate carboxyl group, the phosphorylium group, and the hydrolytic water molecule), other enzymefunctional groups at the active site may be involved as well, or alternatively. In any case, as a natural outcome of their participation in assisting the displacement reaction, the $\mathrm{K}^{+}$ ions are bound to the enzyme either directly or indirectly via the bound phosphate ion. The cleft then reopens in the absence of forces sufficient to hold it closed, and the remaining products of the reaction, inorganic phosphate, water protons, and $\mathrm{K}^{+}$are released to the aqueous medium on the near side prior to the initiation of the next round of catalysis. Before leaving the subject of the ATPases, it should be pointed out that models for the mechanisms of other ATPases, such as the $\mathrm{H}^{+} / \mathrm{K}^{+}$-translocating ATPase of gastric mucosa, the organellar $\mathrm{H}^{+}$-ATPases, the $\mathrm{K}^{+}$-ATPase of $E$. coli, the various $\mathrm{Ca}^{2+} / \mathrm{H}^{+}$-ATPases, and the fungal or plant $\mathrm{H}^{+}$-ATPases or sarcoplasmic reticulum $\mathrm{Ca}^{2+}$-ATPase operating with a counter flux of $\mathrm{K}^{+}$(if indeed any of them ever do), can be readily formulated by using the appropriate parts of Fig. 2, schemes D to G.

The mechanisms of certain of the proton pumps driven by electron transfer reactions can be visualized in a fashion very similar to the rest of the transporters discussed thus far (Fig. 2H). The condensation reaction is driven by favorable bonding interactions between the transporter and hydrogen carriers such as flavine mononucleotide, ubiquinone, etc., designated in Fig. $2 \mathrm{H}$ as a class by the symbol $\mathrm{XH}_{2}$. After the condensation reaction (stages 1 and 2), the displacement reaction ensues with the extraction of two protons from the hydrogen carrier, aided by proton withdrawing groups symbolized as B's, leaving behind four electrons (large dots), two of which flow (small arrows) to an appropriate electron acceptor and two of which remain associated with the former hydrogen carrier (stage 3 ). With little remaining impetus to stay closed, the cleft reopens to the near side, leaving the two protons, which have since departed from the B's and become hydrated, on the far side. The hydrogen carrier is then recharged with two electrons and two protons on the near side in a separate reaction (not shown) whereupon another transport cycle can begin.

In the final scheme of figure 2 , a plausible direct coupling mechanism for the light-driven proton pump, bacteriorhodopsin, is presented. Information as to the chemical reactions that occur in this cycle is incomplete (172-174), and therefore this scheme must be considered as even more approximate than the others that have been proposed up to this point. Nevertheless, even in the absence of chemical detail, this system is of interest in the context of the present article because it provides an opportunity to consider another type of particle flow coupling, through which a flow of photons is utilized for the production of a transmembrane flow of protons against an electrochemical protonic potential difference. This system is also especially pertinent to the present discussion because the specific manner in which the particle flows might be directly coupled is particularly easy to see. As depicted in stages 1 and 2, the condensation reaction is driven by favorable bonding interactions between the transporter and the chromophore, retinal, covalently bound via a protonated Schiff base linkage and in a specific chemical state, designated as $\mathrm{R} 1$. As a result of the condensation reaction, the active site wherein the protonated Schiff base resides, has become in contact with the aqueous medium on the far side, either directly or via a protonconducting channel. The displacement reaction then begins with the absorption of a photon by the retinal moiety. This leads to a change in the chemical state of the retinal moiety (isomerization?) to a new chemical state, designated as R2, and a change in the $\mathrm{pK}_{\mathrm{a}}$ of the Schiff base, such that its deprotonation is favored. The liberated proton diffuses into the aqueous medium on the far side, and in the absence of the favorable bonding interactions between residues in the cleft and the R1 form of the chromophore, the cleft reopens to the near side (stage 4), whereupon the R2 form changes back to the R1 form and the Schiff base is reprotonated, 
again, either directly or via another proton-conducting channel from the near side to the active site, in preparation for another round of catalysis. As drawn, the stoichiometry of the reaction is one proton per cycle, which is at present controversial $(172,174)$. However, it is straightforward to imagine that the changes undergone by the retinal upon the absorption of a photon could affect the $\mathrm{pK}_{\mathrm{a}}$ of one or more other protonated groups in the vicinity of the retinal in addition to the Schiff base, which would account for stoichiometries $>1$.

The general scheme of Fig. $2 \mathrm{I}$ is also readily adapted to explain the mechanism of the light-driven chloride pump, halorhodopsin (162), by simply deemphasizing the protonbinding site(s) and replacing it with a chloride-binding site, which binds chloride tightly when the retinal is in the R2 form and loosely after the retinal changes to the R1 form. Also, for this pump, the active site is likely to be accessible directly from the aqueous medium on each side of the membrane at the appropriate point in the cycle rather than via $\mathrm{Cl}^{-}$conducting channels.

Finally, Fig. 2I can also be used as an aid to visualize how a respiratory chain constituent such as cytochrome $c$ oxidase might translocate protons, if indeed it does $(18,126,130$, $170,182,188-191)$. In this case, the condensation reaction would be driven by favorable bonding interactions between the transporter and a heme group (instead of a retinal) near which a proton is preferentially bound as a result of the oxidation state of the heme iron. Two potential candidates for the proton-binding site that have been suggested are a Schiff base linkage involving a heme formyl group (18) and an axial heme iron ligand $(189,190)$. In any case, after the condensation reaction, which renders the protonated group accessible from the far side, either directly or via a protonconducting channel, a change in the oxidation state of the heme iron as a result of electron transfer, i.e., the displacement reaction, would reduce the affinity of the protonbinding site for its proton and at the same time diminish the forces that hold the cleft closed. This would facilitate proton debinding to the far side and reopening of the cleft to the near side, whereupon the oxidation state of the heme would be changed back as a result of the reciprocal electron transfer reaction, the proton-binding site affinity would be concomitantly increased resulting in its reprotonation, and another pump cycle would then ensue.

For the sake of completeness, it should be mentioned that a discussion of the bacterial binding protein-dependent transport systems $(1,193)$ has been eschewed because the nature of the energy-coupling steps that occur in these systems is as yet unknown.

\section{CONCLUSION}

It is manifestly clear that the task of transporter molecules is to somehow provide a means by which binding sites for the ligands that they transport alternately become exposed to the aqueous media on either side of the membrane through which the transport catalysis occurs. By virtue of the fact that the once-viable alternative, the mobile carrier, is now recognized as unlikely to be operant $(27,90)$ except in the case of very small nonproteinaceous porters such as valinomycin and nigericin, it is now generally acknowledged that conformational changes in more or less fixed, membrane-spanning transporter molecules must constitute the means by which the alternating binding site access is achieved. However, it is in regard to the causal relationship between ligand binding and the protein conformational changes, and, in the cases where the flows of more than one particle are involved, how the particle flows are coupled, that opinions can be found to vary significantly. If there is a majority view $(64,180,183)$, it would appear to be that binding of a transported ligand to more or less preformed sites occurs in a conceptually and physically distinct process that is followed by a conformational change in the transporter molecule which results in translocation of the binding site, with ligand attached, from access at one side of the membrane to access at the other. Furthermore, when the energy of a second particle flow is exerted on the process, it is exerted from a distant site through the transporter molecule and has the effect of changing the affinity of the binding site so that ligand accumulation occurs on one side or the other. An alternative view, presented in this article, is that ligand-binding sites are not preformed and that the conformational transition that brings about the access change is driven and stabilized by the ligand-binding reaction itself, in a process generically termed the condensation reaction. The favorable bonding interactions that stabilize the resulting conformational state of the transporter are then weakened in an ensuing displacement reaction, which facilitates the converse access change, and return of the transporter to the conformational state favored in the absence of ligands. The energy of additional particle flows, as proposed explicitly in Fig. 2, is exerted entirely via residues in or near the respective active sites, and it is the specific chemical events that occur at the active site during the condensation and displacement reactions that determine the species that actually traverse the membrane barrier.

The time is coming, possibly very soon, when we will begin to obtain static glimpses of transporters as they exist at different stages of their transport cycles. This can be anticipated with some confidence because the art of membrane protein crystallization is rapidly developing $(26,28,29,42$, $45,46,56,71,105-109,140-143,169)$, and it would appear to be only a matter of time before high-resolution X-ray crystallographic analyses of the structures of membrane proteins will become routine. As happened in the field of enzymatic catalysis, as these glimpses begin to accumulate, the wealth of biochemical information already generated for transporters will assume its fullest value, and kinematic transport pictures including the relative positions of the atoms of the transported ligands, their binding sites, and the membrane, and in the case of accumulative transporters, the sites of interaction between the transporter and the particles that drive the accumulation as well, will begin to take shape. At that time, if it eventuates for any transporter that the transporter does not undergo its access changes concomitant with ligand binding and displacement, or that the binding site for the transported ligand is physically separated from the site via which a second particle flow occurs, by more than a few angstroms, then it will have to be acknowledged for that transporter that a mechanism of the type proposed in this article is untenable and that an indirect mechanism of the general nature described above is more likely. If, however, evidence for such indirect mechànisms is not forthcoming, and viable alternatives are actively sought, it is hoped that this article might serve to direct attention to the active site, because it is entirely possible that therein may occur most, if not all, of the key reactions in the membrane transport process.

\section{ACKNOWLEDGMENT}

This work was supported by Public Health Service grant GM24784 from the National Institutes of Health. 


\section{LITERATURE CITED}

1. Ames, G. F.-L., and C. F. Higgins. 1983. The organization, mechanism of action, and evolution of periplasmic transport systems. Trends Biochem. Sci. 8:97-100.

2. Amzel, L. M., and P. L. Pedersen. 1983. Proton ATPases: structure and mechanism. Annu. Rev. Biochem. 52:801-824.

3. Anderson, C. M., F. H. Zucker, and T. A. Steitz. 1979. Space-filling models of kinase clefts and conformation changes. Science 204:375-380.

4. Bamberg, E., N. A. Dencher, A. Fahr, and M. P. Heyn. 1981. Transmembraneous incorporation of photoelectrically active bacteriorhodopsin in planar lipid bilayers. Proc. Natl. Acad. Sci. U.S.A. 78:7502-7506.

5. Beers, M. F., S. E. Carty, R. G. Johnson, and A. Scarpa. 1982. $\mathrm{H}^{+}$-ATPase and catecholamine transport in chromaffin granules. Ann. N.Y. Acad. Sci. 402:116-133.

6. Begley, G. S., D. E. Hansen, G. R. Jacobson, and J. R. Knowles. 1982. Stereochemical course of the reactions catalyzed by the bacterial phosphoenolpyruvate: glucose phosphotransferase system. Biochemistry 21:5552-5556.

7. Bhattacharyya, P., and E. M. Barnes, Jr. 1976. ATP-dependent calcium transport in isolated membrane vesicles from Azotobacter vinelandii. J. Biol. Chem. 251:5614-5619.

8. Blaustein, M. P. 1984. The energetics and kinetics of sodiumcalcium exchange in barnacle muscles, squid axons, and mammalian heart: the role of ATP, p. 129-147. In M. P. Blaustein and M. Lieberman (ed.), Electrogenic transport: fundamental principles and physiological implications. Raven Press, New York.

9. Blaustein, M. P., and M. T. Nelson. 1982. Sodium-calcium exchange: its role in the regulation of cell calcium, p. 217-236. In E. Carafoli (ed.), Membrane transport of calcium. Academic Press, Inc., New York.

10. Boyer, P. D., W. E. Kohlbrenner, D. B. McIntosh, L. T. Smith, and C. C. O'Neal. 1982. ATP and ADP modulations of catalysis of $\mathrm{F}_{1}$ and $\mathrm{Ca}^{2+}, \mathrm{Mg}^{2+}$-ATPases. Ann. N.Y. Acad. Sci. 402:65-83.

11. Brey, R. N., and B. P. Rosen. 1979. Cation/proton antiport systems in Escherichia coli: properties of the calcium/proton antiporter. J. Biol. Chem. 254:1957-1963.

12. Brotherus, J. R., J. V. Moller, and P. L. Jorgensen. 1981. Soluble and active renal Na, K-ATPase with maximum protein molecular mass $170,000 \pm 9,000$ daltons; formation of larger units by secondary aggregation. Biochem. Biophys. Res. Commun. 100:146-154.

13. Bürkler, J., and M. Solioz. 1982. The ATP-dependent $\mathrm{Ca}^{2+}$ pumping system of Streptococcus faecium. Ann. N.Y. Acad. Sci. 402:422-432.

14. Carafoli, E., M. Zurini, V. Niggli, and J. Krebs. 1982. The calcium-transporting ATPase of erythrocytes. Ann. N.Y. Acad. Sci. 402:304-328.

15. Changeux, J.-P., A. Devillers-Thiéry, and P. Chemouilli. 1984. Acetylcholine receptor: an allosteric protein. Science 225:1335-1345

16. Citri, N. 1973. Conformational adaptability in enzymes. Adv. Enzymol. 37:397-648.

17. Clarke, S. 1975 . The size and detergent binding of membrane proteins. J. Biol. Chem. 250:5459-5469.

18. Coin, J. T., and P. C. Hinkle. 1979. Proton transport by reconstituted segments of the respiratory chain, p. 405-412. In C. P. Lee, G. Schatz, and L. Ernster (ed.), Membrane bioenergetics. Addison-Wesley, Reading, Mass.

19. Conti-Tronconi, B. M., and M. A. Raftery. 1982. The nicotinic cholinergic receptor: correlation of molecular structure with functional properties. Annu. Rev. Biochem. 51:491-530.

20. Crane, R. K. 1965 . $\mathrm{Na}^{+}$-dependent transport in the intestine and other animal tissues. Fed. Proc. 24:1000-1006.

21. Cross, R. L. 1981. The mechanism and regulation of ATP synthesis by $F_{1}$-ATPases. Annu. Rev. Biochem. 50:681-714.

22. Dame, J. B., and G. A. Scarborough. 1981. Identification of the phosphorylated intermediate of the Neurospora plasma membrane $\mathrm{H}^{+}$-ATPase as $\beta$-aspartyl phosphate. J. Biol. Chem.
256:10724-10730

23. Dean, W. L., and C. Tanford. 1978. Properties of a delipidated, detergent-activated $\mathrm{Ca}^{2+}$-ATPase. Biochemistry 17:1683-1690.

24. deMeis, L., and A. L. Vianna. 1979. Energy interconversion by the $\mathrm{Ca}^{2+}$-dependent ATPase of the sarcoplasmic reticulum. Annu. Rev. Biochem. 48:275-292.

25. Dencher, N. A., and M. P. Heyn. 1979. Bacteriorhodopsin monomers pump protons. FEBS Lett. 108:307-310.

26. Dorset, D. L., A. Engel, M. Haner, A. Massalski, and J. P. Rosenbusch. 1983. Two-dimensional crystal packing of matrix porin, a channel forming protein in Escherichia coli outer membranes. J. Mol. Biol. 165:701-710.

27. Dutton, A., E. D. Rees, and S. J. Singer. 1976. An experiment eliminating the rotating carrier mechanism for the active transport of $\mathrm{Ca}$ ion in sarcoplasmic reticulum membranes. Proc. Natl. Acad. Sci. U.S.A. 73:1532-1536.

28. Dux, L., and A. Martonosi. 1983. Two-dimensional arrays of proteins in sarcoplasmic reticulum and purified $\mathrm{Ca}^{2+}$-ATPase vesicles treated with vanadate. J. Biol. Chem. 258:2599-2603.

29. Dux, L., and A. Martonosi. 1983. $\mathrm{Ca}^{2+}$-ATPase membrane crystals in sarcoplasmic reticulum: the effect of trypsin digestion. J. Biol. Chem. 258:10111-10115.

30. Eddy, A. A. 1980. Slip and leak models of gradient-coupled solute transport. Biochem. Soc. Trans. 8:271-273.

31. Eklund, H., B. Nordström, E. Zeppezauer, G. Söderlund, I. Ohlsson, T. Boiwe, B.-O. Söderburg, O. Tapia, and C.-I. Brändén. 1976. Three-dimensional structure of horse liver alcohol dehydrogenase at $2.4 \AA$ resolution. J. Mol. Biol. 102:27-59.

32. Eklund, H., J. P. Samama, L. Wallén, and C.-I. Brändén. 1981. Structure of a triclinic ternary complex of horse liver alcohol dehydrogenase at 2.9 ̊ resolution. J. Mol. Biol. 146:561-587.

33. Epstein, W., V. Whitelaw, and J. Hesse. 1978. A K ${ }^{+}$transport ATPase in Escherichia coli. J. Biol. Chem. 253:6666-6668.

34. Eyring, H., R. Lumry, and J. W. Woodbury. 1949. Some applications of modern rate theory to physiological systems. Rec. Chem. Prog. 10:100-114.

35. Faller, L., R. Jackson, D. Malinowska, E. Mukidjam, E. Rabon, G. Saccomani, G. Sachs, and A. Smolka. 1982. Mechanistic aspects of gastric $\left(\mathrm{H}^{+}+\mathrm{K}^{+}\right)$-ATPase. Ann. N.Y. Acad. Sci. 402:146-163.

36. Feldman, R. I., and D. S. Sigman. 1983. The synthesis of ATP by the membrane-bound ATP synthase complex from medium ${ }^{32} \mathrm{Pi}$ under completely uncoupled conditions. J. Biol. Chem. 258:12178-12183.

37. Ferguson, S. J., and M. C. Sorgato. 1982. Proton electrochemical gradients and energy-transduction processes. Annu. Rev. Biochem. 51:185-217.

38. Fillingame, R. H. 1980 . The proton-translocating pumps of oxidative phosphorylation. Annu. Rev. Biochem. 49:1079 1113.

39. Forgac, M., L. Cantley, B. Wiedenmann, L. Altstiel, and D. Branton. 1983. Clathrin-coated vesicles contain an ATPdependent proton pump. Proc. Natl. Acad. Sci. U.S.A. 80:1300-1303.

40. Frost, A. A., and R. G. Pearson. 1961. Kinetics and mechanism, 2nd ed. John Wiley \& Sons, Inc., New York.

41. Fukada, H., J. M. Sturtevant, and F. A. Quiocho. 1983. Thermodynamics of the binding of L-arabinose and of Dgalactose to the L-arabinose-binding protein of Escherichia coli. J. Biol. Chem. 258:13193-13198.

42. Fuller, S. D., R. A. Capaldi, and R. Henderson. 1979. Structure of cytochrome c oxidase in deoxycholate-derived twodimensional crystals. J. Mol. Biol. 134:305-327.

43. Futai, M., and H. Kanazawa. 1983. Structure and function of proton-translocating adenosine triphosphatase $\left(\mathrm{F}_{\mathrm{o}} \mathrm{F}_{1}\right)$ : biochemical and molecular biological approaches. Microbiol. Rev. 47:285-312.

44. Gabizon, R., and S. Schuldiner. 1985 . The amine transporter from bovine chromaffin granules: partial purification. J. Biol. Chem. 260:3001-3005.

45. Garavito, R. M., U. Hinz, and J.-M. Neuhaus. 1984. The crystallization of outer membrane proteins from Escherichia 
coli: studies on lam B and omp A gene products. J. Biol. Chem. 259:4254-4257.

46. Garavito, R. M., J. Jenkins, J. N. Jansonius, R. Karlsson, and J. P. Rosenbusch. 1983. X-ray diffraction analysis of matrix porin, an integral membrane protein from Escherichia coli outer membranes. J. Mol. Biol. 164:313-327.

47. Gilliland, G. L., and F. A. Quiocho. 1981. Structure of the L-arabinose-binding protein from Escherichia coli at $2.4 \AA$ resolution. J. Mol. Biol. 146:341-362.

48. Glynn, I. M., and S. J. D. Karlish. 1975. The sodium pump. Annu. Rev. Physiol. 37:13-55.

49. Goffeau, A., and C. W. Slayman. 1981. The protontranslocating ATPase of the fungal plasma membrane. Biochim. Biophys. Acta 639:197-223.

50. Govindjee (ed.). 1982. Photosynthesis: energy conversion by plants and bacteria. Academic Press, Inc., New York.

51. Grisham, C. M. 1981. Characterization of ATP binding sites of sheep kidney medulla $\left(\mathrm{Na}^{+}+\mathrm{K}^{+}\right)$-ATPase using CrATP. J. Inorg. Biochem. 14:45-57.

52. Grisham, C. M., R. K. Gupta, R. E. Barnett, and A. S. Mildvan. 1974. Thallium-205 nuclear relaxation and kinetic studies of sodium and potassium ion-activated adenosine triphosphatase. J. Biol. Chem. 249:6738-6744.

53. Grisham, C. M., and A. S. Mildvan. 1975. Magnetic resonance and kinetic studies of the mechanism of membrane-bound sodium and potassium ion-activated adenosine triphosphatase. J. Supramol. Struct. 3:304-313.

54. Harrison, S. C. 1978. Structure of simple viruses: specificity and flexibility in protein assemblies. Trends Biochem. Sci. 3:3-7.

55. Harrison, S. C. 1980. Protein interfaces and intersubunit bonding: the case of tomato bushy stunt virus. Biophys. J. 32:139-151.

56. Hebert, H., P. L. Jorgensen, E. Skriver, and A. B. Maunsbach. 1982. Crystallization patterns of membrane-bound $\left(\mathrm{Na}^{+}+\mathrm{K}^{+}\right)$ ATPase. Biochim. Biophys. Acta 689:571-574.

57. Hesse, J. E., L. Wieczorek, K. Altendorf, A. S. Reicin, E. Dorus, and W. Epstein. 1984. Sequence homology between two membrane transport ATPases, the Kdp-ATPase of Escherichia coli and the $\mathrm{Ca}^{2+}$-ATPase of sarcoplasmic reticulum. Proc. Natl. Acad. Sci. U.S.A. 81:4746-4750.

58. Hill, T. L. 1977. Free energy transduction in biology. Academic Press, Inc., New York.

59. Hopfer, U., and R. Groseclose. 1980. The mechanism of $\mathrm{Na}^{+}$dependent D-glucose transport. J. Biol. Chem. 255:4453-4462.

60. Huber, R. 1979. Conformational flexibility and its functional significance in some protein molecules. Trends Biochem. Sci. 4:271-276

61. Hüdig, H., and W. Hengstenberg. 1980. The bacterial phosphoenolpyruvate dependent phosphotransferase system (PTS): solubilization and kinetic parameters of the glucosespecific membrane bound enzyme II component of Streptococcus faecalis. FEBS Lett. 114:103-106.

62. Imoto, T., L. N. Johnson, A. C. T. North, D. C. Phillips, and J. A. Rupley. 1972. Vertebrate lysozymes. Enzymes 7: 665-868.

63. Janin, J., and S. J. Wodak. 1983. Structural domains in proteins and their role in the dynamics of protein function. Prog. Biophys. Mol. Biol. 42:21-78.

64. Jardetzky, O. 1966. Simple allosteric model for membrane pumps. Nature (London) 211:969-970.

65. Jencks, W. P. 1966. Strain and conformation change in enzymatic catalysis, p. 273-298. In N. O. Kaplan and E. P. Kennedy (ed.), Current aspects of biochemical energetics. Academic Press, Inc., New York.

66. Jennings, M. L. 1984. Oligomeric structure and the anion transport function of human erythrocyte band 3 protein. $J$. Membr. Biol. 80:105-117.

67. Johnson, R. G., D. Pfister, S. E. Carty, and A. Scarpa. 1979. Biological amine transport in chromaffin ghosts: coupling to the transmembrane proton and potential gradients. J. Biol. Chem. 254:10963-10972.

68. Johnson, R. G., and A. Scarpa. 1984. Chemiosmotic coupling and its application to the accumulation of biological amines in secretory granules, p. 71-91. In M. P. Blaustein and M. Lieberman (ed.), Electrogenic transport: fundamental principles and physiological implications. Raven Press, New York.

69. Jones, M. N., and J. K. Nickson. 1980. Identifying the monosaccharide transport protein in the human erythrocyte membrane. FEBS Lett. 115:1-8.

70. Jorgensen, P. L. 1982. Mechanism of the $\mathrm{Na}^{+}, \mathrm{K}^{+}$pump: protein structure and conformations of the pure $\left(\mathrm{Na}^{+}+\right.$ $\mathrm{K}^{+}$)-ATPase. Biochim. Biophys. Acta 694:27-68.

71. Jorgensen, P. L., E. Skriver, H. Hebert, and A. B. Maunsbach. 1982. Structure of the $\mathrm{Na}, \mathrm{K}$ pump: crystallization of pure membrane-bound $\mathrm{Na}$, K-ATPase and identification of functional domains of the $\alpha$-subunit. Ann. N.Y. Acad. Sci. 402:207-225.

72. Jung, C. Y. 1975. Carrier-mediated glucose transport across human red cell membranes, p. 705-751. In D. M. Surgenor (ed.), The red blood cell, vol. 2. Academic Press, Inc., New York.

73. Kaback, H. R. 1968. The role of the phosphoenolpyruvatephosphotransferase system in the transport of sugars by isolated membrane preparations of Escherichia coli. J. Biol. Chem. 243:3711-3724

74. Kaback, H. R. 1983. The lac carrier protein in Escherichia coli. J. Membr. Biol. 76:95-112.

75. Kagawa, Y., N. Sone, H. Hirata, and M. Yoshida. 1979. Structure and function of $\mathrm{H}^{+}$-ATPase. J. Bioenerg. Biomembr. 11:39-78.

76. Kanner, B. I., H. Fishkes, R. Maron, I. Sharon, and S. Schuldiner. 1979. Reserpine as a competitive and reversible inhibitor of the catecholamine transporter of bovine chromaffin granules. FEBS Lett. 100:175-178.

77. Karplus, M., and J. A. McCammon. 1983. Dynamics of proteins: elements and function. Annu. Rev. Biochem. 52:263300 .

78. Kasahara, M., and P. C. Hinkle. 1977. Reconstitution and purification of the D-glucose transporter from human erythrocytes. J. Biol. Chem. 252:7384-7390.

79. Khananshvili, D., and Z. Gromet-Elhanan. 1985. Evidence that the Mg-dependent low-affinity binding site for ATP and Pi demonstrated on the isolated $\beta$ subunit of the F0F1 ATP synthase is a catalytic site. Proc. Natl. Acad. Sci. U.S.A. 82:1886-1890.

80. Kimmich, G. A., and C. Carter-Su. 1978. Membrane potentials and the energetics of intestinal $\mathrm{Na}^{+}$-dependent transport systems. Am. J. Physiol. 253:C73-C81.

81. King, E. L., and C. Altman. 1956. A schematic method of deriving the rate laws for enzyme-catalyzed reactions. J. Phys. Chem. 60:1375-1378.

82. Klingenberg, M. 1981. Membrane protein oligomeric structure and transport function. Nature (London) 290:449-454.

83. Klingenberg, M., H. Aquila, R. Kramer, W. Babel, and J. Feckl. 1977. The ADP, ATP translocation and its catalyst, p. 567-579. In G. Semenza and E. Carafoli (ed.), Biochemistry of membrane transport. Springer-Verlag, New York.

84. Klingenberg, M., I. Mayer, and A. S. Dahms. 1984. Interaction of fluorescent adenine nucleotide derivatives with the ADP/ATP carrier in mitochondria 1. Comparison of various 3'-O-ester adenine nucleotide derivatives. Biochemistry 23:2436-2442.

85. Knowles, J. R. 1980. Enzyme-catalyzed phosphoryl transfer reactions. Annu. Rev. Biochem. 49:877-919.

86. Konings, W. N., and I. R. Booth. 1981. Do the stoichiometries of ion-linked transport systems vary? Trends Biochem. Sci. 2:257-262

87. Koshland, D. E., Jr., and K. E. Neet. 1968. The catalytic and regulatory properties of enzymes. Annu. Rev. Biochem. 37: $359-410$.

88. Kozlov, I. A., and V. P. Skulachev. 1977. $\mathrm{H}^{+}$-adenosine triphosphatase and membrane energy coupling. Biochim. Biophys. Acta 463:29-89.

89. Krulwich, T. A. $1983 . \mathrm{Na}^{+} / \mathrm{H}^{+}$antiporters. Biochim. Biophys. Acta 726:245-264. 
90. Kyte, J. 1974. The reactions of sodium and potassium ionactivated adenosine triphosphatase with specific antibodies. J. Biol. Chem. 249:3652-3660.

91. Kyte, J. 1981. Molecular considerations relevant to the mechanism of active transport. Nature (London) 292:201-204.

92. Läuger, P. 1980. Kinetic properties of ion carriers and channels. J. Membr. Biol. 57:163-178.

93. LeFevre, P. G. 1975. A comparison of recent suggestions for the functional organization of red-cell sugar-transport sites based on kinetic observations. Ann. N.Y. Acad. Sci. 264: 398-413.

94. Lienhard, G. E. 1973. Enzymatic catalysis and transition-state theory. Science 180:149-154.

95. Lipmann, F. 1960. Attempts toward a formulation of biological use of energy in terms of chemical potentials, p. 37-47. In D. Nachmansohn (ed.), Molecular biology. Academic Press, Inc., New York.

96. Lowe, A. G., and A. Lambert. 1983. Chloride-bicarbonate exchange and related transport processes. Biochim. Biophys. Acta 694:353-374.

97. Maloney, P. C. 1982. Energy coupling to ATP synthesis by the proton-translocating ATPase. J. Membr. Biol. 67:1-12.

98. Mao, B., and J. A. McCammon. 1983. Theoretical study of hinge-bending in L-arabinose-binding protein: internal energy and free energy changes. J. Biol. Chem. 258:12543-12547.

99. Mao, B., and J. A. McCammon. 1984. Structural study of hinge bending in L-arabinose-binding protein. J. Biol. Chem. 259:4964-4970.

100. Mao, B., M. R. Pear, J. A. McCammon, and F. A. Quiocho. 1982. Hinge-bending in $\mathrm{L}$-arabinose-binding protein: the Venus's-flytrap model. J. Biol. Chem. 257:1131-1133.

101. Martin, D. W., C. Tanford, and J. A. Reynolds. 1984. Monomeric solubilized sarcoplasmic reticulum Ca pump protein: demonstration of $\mathrm{Ca}$ binding and dissociation coupled to ATP hydrolysis. Proc. Natl. Acad. Sci. U.S.A. 81:6623-6626.

102. McCammon, J. A., and M. Karplus. 1977. Internal motions of antibody molecules. Nature (London) 268:765-766.

103. McEnery, M. W., E. L. Buhle, Jr., U. Aebi, and P. L. Pedersen. 1984. Proton ATPase of the rat liver mitochondria: preparation and visualization of a functional complex using the novel zwitterionic detergent 3-((3-cholamidopropyl)dimethylammonio)-1-propanesulfonate. J. Biol. Chem. 259:4642-4651.

104. Michalak, M., K. Famulski, and E. Carafoli. 1984. The $\mathrm{Ca}^{2+}$ pumping ATPase in skeletal muscle sarcolemma: calmodulin dependence, regulation by cAMP-dependent phosphorylation, and purification. J. Biol. Chem. 259:15540-15547.

105. Michel, H. 1982. Three-dimensional crystals of a membrane protein complex: the photosynthetic reaction centre from Rhodopseudomonas viridis. J. Mol. Biol. 158:567-572.

106. Michel, H. 1982. Characterization and crystal packing of threedimensional bacteriorhodopsin crystals. EMBO J. 1:12671271.

107. Michel, H. 1983. Crystallization of membrane proteins. Trends Biochem. Sci. 8:56-59.

108. Michel, H., and D. Oesterhelt. 1980. Three-dimensional crystals of membrane proteins: bacteriorhodopsin. Proc. Natl Acad. Sci. U.S.A. 77:1283-1285.

109. Michel, H., D. Oesterhelt, and R. Henderson. 1980 Orthorhombic two-dimensional crystal form of purple membrane. Proc. Natl. Acad. Sci. U.S.A. 77:338-342.

110. Miller, D. M., III, J. S. Olson, J. W. Pflugrath, and F. A. Quiocho. 1983. Rates of ligand binding to periplasmic proteins involved in bacterial transport and chemotaxis. J. Biol. Chem. 258:13665-13672.

111. Missett, O., M. Blaauw, P. W. Postma, and G. T. Robillard. 1983. Bacterial phosphoenolpyruvate-dependent phosphotransferase system. Mechanism of the transmembrane sugar translocation and phosphorylation. Biochemistry 22:61636170.

112. Mitchell, P. 1957. A general theory of membrane transport from studies of bacteria. Nature (London) 180:134-136.

113. Mitchell, P. 1963. Molecule, group and electron translocation through natural membranes. Biochem. Soc. Symp. 22:142-169.
114. Mitchell, P. 1967. Active transport and ion accumulation, p. 167-197. In M. Florkin and E. H. Stotz (ed.), Comprehensive biochemistry, vol. 22. Elsevier, Amsterdam.

115. Mithcell, P. 1969. Chemiosmotic coupling and energy transduction. Theor. Exp. Biophys. 2:159-216.

116. Mitchell, P. 1973. Cation-translocating adenosine triphosphatase models: how direct is the participation of adenosine triphosphate and its hydrolysis products in cation translocation? FEBS Lett. 33:267-274.

117. Mitchell, P. 1974. A chemiosmotic molecular mechanism for proton-translocating adenosine triphosphatases. FEBS Lett. 43:189-194.

118. Mitchell, P. 1975. Proton translocation mechanisms and energy transduction by adenosine triphosphatases: an answer to criticisms. FEBS Lett. 50:95-97.

119. Mitchell, P. 1977. Epilogue: from energetic abstraction to biochemical mechanism. Symp. Soc. Gen. Microbiol. 27: 383-423.

120. Mitchell, P. 1979. Direct chemiosmotic ligand conduction mechanisms in protonmotive complexes, p. 361-372. In C. P. Lee, G. Schatz, and L. Ernster (ed.), Membrane bioenergetics. Addison-Wesley, Reading Mass.

121. Mitchell, P. 1979. Compartmentation and communication in living systems. Ligand conduction: a general catalytic principle in chemical, osmotic and chemiosmotic reaction systems. Eur. J. Biochem. 95:1-20.

122. Mitchell, P. 1980. The culture of imagination. An essay on creativity in the sciences, in the arts, and in life. J. R. Inst. Cornwall New Ser. 8:173-191.

123. Mitchell, P. 1981. Bioenergetic aspects of unity in biochemistry: evolution of the concept of ligand conduction in chemical, osmotic, and chemiosmotic reaction mechanisms, p. 1-160. In G. Semenza (ed.), Of oxygen, fuels, and living matter, part 1. John Wiley \& Sons, Chichester, England.

124. Mitchell, P. 1981. From black-box bioenergetics to molecular mechanics: vectorial ligand-conduction mechanisms in biochemistry, p. 611-631. In V. P. Skulachev and P. C. Hinkle (ed.), Chemiosmotic proton circuits in biological membranes. Addison-Wesley, Reading, Mass.

125. Mitchell, P. 1981. Biochemical mechanism of protonmotivated phosphorylation in F0F1 adenosine triphosphatase molecules, p. 427-457. In C. P. Lee, G. Schatz, and G. Dallner (ed.), Mitochondria and microsomes. Addison-Wesley, Reading, Mass.

126. Mitchell, P. 1981. Ubiquinone, bioenergetics and still point of the turning cycle, p. 3-15. In K. Folkers and Y. Yamamura (ed.), Biomedical and clinical aspects of coenzyme Q, vol. 3. Elsevier/North-Holland Biomedical Press, Amsterdam.

127. Mitchell, P., and W. H. Koppenol. 1982. Chemiosmotic ATPase mechanisms. Ann. N.Y. Acad. Sci. 402:584-601.

128. Mitchell, P., and J. Moyle. 1958. Group-translocation: a consequence of enzyme-catalyzed group-transfer. Nature (London) 182:372-373.

129. Mitchell, P., and J. Moyle. 1974. The mechanism of proton translocation in reversible proton-translocating adenosine triphosphatases. Biochem. Soc. Spec. Publ. 4:91-111.

130. Mitchell, P., and J. Moyle. 1983. Alternative hypotheses of proton ejection in cytochrome oxidase vesicles. Transmembrane proton pumping or redox-linked deprotonation of phospholipid-cytochrome c complex(es). FEBS Lett. 151: $167-178$

131. Neuhaus, J.-M. 1982. The receptor protein of phage $\lambda$ : purification, characterization and preliminary electrical studies in planar lipid bilayers. Ann. Microbiol. 133A:27-32.

132. Newcomer, M. E., G. L. Gilliland, and F. A. Quiocho. 1981 $\mathrm{L}$-arabinose-binding protein-sugar complex at $2.4 \AA$ resolution stereochemistry and evidence for a structural change. J. Biol. Chem. 256:13213-13217.

133. Newcomer, M. E., B. A. Lewis, and F. A. Quiocho. 1981. The radius of gyration of $\mathrm{L}$-arabinose-binding protein decreases upon binding of ligand. J. Biol. Chem. 256:13218-13222.

134. Newcomer, M. E., D. M. Miller III, and F. A. Quiocho. 1979. Location of the sugar-binding site of L-arabinose-binding pro- 
tein: sugar derivative syntheses, sugar binding specificity, and difference Fourier analyses. J. Biol. Chem. 254:7529-7533.

135. Northrup, S. H., F. Zarrin, and J. A. McCammon. 1982. Rate theory for gated diffusion-influenced ligand binding to proteins. J. Phys. Chem. 86:2314-2321.

136. Ohsumi, Y., and Y. Anraku. 1981. Active transport of basic amino acids driven by a proton motive force in vacuolar membrane vesicles of Saccharomyces cerevisiae. J. Biol. Chem. 256:2079-2082.

137. Ovchinnikov, Y. A., N. G. Abdulaev, and N. N. Modyanov. 1982. Structural basis of proton-translocating protein function. Annu. Rev. Biophys. Bioeng. 11:445-463.

138. Overath, P., and J. K. Wright. 1983. Lactose permease: a carrier on the move. Trends Biochem. Sci. 8:404-408.

139. Overton, E. 1899. Ueber die allgemeinen osmotischen Eigenschaften der Zelle, ihre vermutlichen Ursachen und ihre Bedeutung für die Physiologie. Vierteljahrschr. Naturforsch. Ges. Zuerich 44:88-135.

140. Ozawa, T., H. Suzuki, and M. Tanaka. 1980. Cyrstallization of part of the mitochondrial electron transfer chain: cytochrome $c$ oxidase-cytochrome c complex. Proc. Natl. Acad. Sci. U.S.A. 77:928-930.

141. Ozawa, T., M. Tanaka, and Y. Shimomura. 1980. Crystallization of the middle part of the mitochondrial electron transfer chain: cytochrome $b c_{1}$-cytochrome c complex. Proc. Natl. Acad. Sci. U.S.A. 77:5084-5086.

142. Ozawa, T., M. Tanaka, and Y. Shimomura. 1983. Crystalliza-

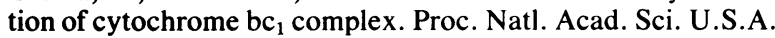
80:921-925.

143. Ozawa, T., M. Tanaka, and T. Wakabayashi. 1982. Crystallization of mitochondrial cytochrome oxidase. Proc. Natl. Acad. Sci. U.S.A. 79:7175-7179.

144. Pauling, L. 1946. Molecular architecture and biological reactions. Chem. Eng. News 24:1375-1377.

145. Perret, J., and P. Gay. 1979. Kinetic study of a phosphoryl exchange reaction between fructose and fructose 1-phosphate catalyzed by the membrane-bound enzyme II of the phosphoenolpyruvate-fructose 1-phosphotransferase system of $\mathrm{Ba}$ cillus subtilis. Eur. J. Biochem. 102:237-246.

146. Poole, R. J. 1978. Energy coupling for membrane transport in plants. Annu. Rev. Plant. Physiol. 29:437-460.

147. Popper, K. R. 1972. The logic of scientific discovery, 3rd (revised) ed. Hutchinson, London.

148. Reeves, J. P., and J. L. Sutko. 1979. Sodium-calcium ion exchange in cardiac membrane vesicles. Proc. Natl. Acad. Sci. U.S.A. 76:590-594.

149. Reider, E., E. F. Wagner, and M. Schweiger. 1979. Control of phosphoenolpyruvate-dependent phosphotransferase-mediated sugar transport in Escherichia coli by energization of the cell membrane. Proc. Natl. Acad. Sci. U.S.A. 76:5529-5533.

150. Remington, S., G. Wiegand, and R. Huber. 1982. Crystallographic refinement and atomic models of two different forms of citrate synthase at 2.7 and $1.7 \AA$ resolution. J. Mol. Biol. 158:111-152.

151. Robillard, G. T. 1982. The enzymology of the bacterial phosphoenolpyruvate-dependent sugar transport systems. Mol. Cell. Biochem. 46:3-24.

152. Rosen, B. P. 1982. Calcium transport in microorganisms, p. 187-216. In E. Carafoli (ed.), Membrane transport of calcium. Academic Press, Inc., New York.

153. Ross, A. H., and H. M. McConnell. 1978. Reconstitution of the erythrocyte anion channel. J. Biol. Chem. 253:4777-4782.

154. Rothstein, A., Z. I. Cabantchik, and P. Knauf. 1976. Mechanism of anion transport in red blood cells: role of membrane proteins. Fed. Proc. 35:3-10.

155. Rothstein, A., P. A. Knauf, S. Grinstein, and Y. Shami. 1979. A model for the action of the anion exchange protein of the red blood cell. Prog. Clin. Biol. Res. 30:483-496.

156. Sachs, G., B. Wallmark, G. Saccomani, E. Rabon, H. B. Stewart, D. R. DiBona, and T. Berglindh. 1982. The ATPdependent component of gastric acid secretion. Curr. Top. Membr. Transp. 16:135-159.

157. Saier, M. H., Jr. 1977. Bacterial phosphoenolpyruvate:sugar phosphotransferase systems: structural, functional, and evolutionary relationshps. Bacteriol. Rev. 41:856-871.

158. Scarborough, G. A. 1982 . The Neurospora plasma membrane ATPase, p. 431-438. In D. Marmé, E. Marrè, and R. Hertel (ed.), Plasmalemma and tonoplast: their functions in the plant cell. Elsevier Biomedical Press B.V., Amsterdam.

159. Scarborough, G. A. 1982. Chemiosmotic models for the mechanisms of the cation-motive ATPases. Ann. N.Y. Acad. Sci. 402:99-115.

160. Schatzmann, H. J. 1983. The red cell calcium pump. Annu Rev. Physiol. 45:303-312.

161. Schindler, H., and J. P. Rosenbusch. 1981. Matrix protein in planar membranes: clusters of channels in a native environment and their functional reassembly. Proc. Natl. Acad. Sci. U.S.A. 78:2302-2306.

162. Schobert, B., and J. K. Lanyi. 1982. Halorhodopsin is a light-driven chloride pump. J. Biol. Chem. 257:10306-10313.

163. Schuldiner, S., H. Fishkes, and B. I. Kanner. 1978. Role of a transmembrane $\mathrm{pH}$ gradient in epinephrine transport by chromaffin granule membrane vesicles. Proc. Natl. Acad. Sci. U.S.A. 75:3713-3716.

164. Schulz, G. E., and R. H. Schirmer. 1979. Principles of protein structure. Springer-Verlag, New York.

165. Schulz, G. E., R. H. Schirmer, W. Sachsenheimer, and E. F Pai. 1978. The structure of the flavoenzyme glutathione reductase. Nature (London) 273:120-124.

166. Semenza, G., M. Kessler, M. Hosang, J. Weber, and U. Schmidt. 1984. Biochemistry of the $\mathrm{Na}^{+}$, D-glucose cotransporter of the small-intestinal brush-border membrane: the state of the art in 1984. Biochim. Biophys. Acta 779: 343-379.

167. Senior, A. E., and J. G. Wise. 1983. The proton-ATPase of bacteria and mitochondria. J. Membr. Biol. 73:105-124.

168. Serrano, R. 1984. Plasma membrane ATPase of fungi and plants as a novel type of proton pump. Curr. Top. Cell. Regul. 23:87-126.

169. Skriver, E., A. B. Maunsbach, and P. L. Jorgensen. 1981. Formation of two-dimensional crystals in pure membranebound $\mathrm{Na}^{+}, \mathrm{K}^{+}$-ATPase. FEBS Lett. 131:219-222.

170. Sone, N., and P. C. Hinkle. 1982. Proton transport by cytochrome c oxidase from the thermophilic bacterium PS3 reconstituted in liposomes. J. Biol. Chem. 257:12600-12604.

171. Stein, W. D. 1967. The movement of molecules across cell membranes. Academic Press, Inc., New York.

172. Stoeckenius, W., and R. A. Bogomolni. 1982. Bacteriorhodopsin and related pigments of halobacteria. Annu. Rev. Biochem. 51:587-616.

173. Stoeckenius, W., R. H. Lozier, and R. A. Bogomolni. 1979. Bacteriorhodopsin and the purple membrane of halobacteria. Biochim. Biophys. Acta 505:215-278.

174. Stoeckenius, W., R. H. Lozier, and R. A. Bogomolni. 1981. Bacteriorhodopsin photocycle and stoichiometry, p. 283-309. In V. P. Skulachev and P. C. Hinkle (ed.), Chemiosmotic proton circuits in biological membranes. Addison-Wesley, Reading, Mass.

175. Stone, D. K., X.-S. Xie, and E. Racker. 1983. An ATP-driven proton pump in clathrin-coated vesicles. J. Biol. Chem. 258:4059-4062.

176. Stroobant, P., J. B. Dame, and G. A. Scarborough. 1980. The Neurospora plasma membrane $\mathrm{Ca}^{2+}$ pump. Fed. Proc. 39:2437-2441.

177. Stroobant, P., and G. A. Scarborough. 1979. Active transport of calcium in Neurospora plasma membrane vesicles. Proc. Natl. Acad. Sci. U.S.A. 76:3102-3106.

178. Tada, M., T. Yamamoto, and Y. Tonomura. 1978. Molecular mechanism of active calcium transport by sarcoplasmic reticulum. Physiol. Rev. 58:1-79.

179. Tanford, C. 1982 . Simple model for the chemical potential change of a transported ion in active transport. Proc. Natl. Acad. Sci. U.S.A. 79:2882-2884.

180. Tanford, C. 1983. Mechanism of free energy coupling in active transport. Annu. Rev. Biochem. 52:379-409.

181. Tanford, C. 1985 . Twenty questions concerning the reaction 
cycle of $\mathrm{t}$ sarcoplasmic reticulum calcium pump. Crit. Rev. Biochem. 17:123-151.

182. Thelen, M., P. S. O'Shea, G. Petrone, and A. Azzi. 1985. Proton translocation by a native and subunit III-depleted cytochrome c oxidase reconstituted into phospholipid vesicles: use of fluorescein-phosphatidylethanolamine as an intravesicular $\mathrm{pH}$ indicator. J. Biol. Chem. 260:3626-3631.

183. Vidaver, G. A. 1966. Inhibition of parallel flux and augmentation of counter flux shown by transport models not involving a mobile carrier. J. Theor. Biol. 10:301-306.

184. Visser, L., N. C. Robinson, and C. Tanford. 1975. The twodomain structure of cytochrome $b_{5}$ in deoxycholate solution. Biochemistry 14:1194-1199.

185. Warncke, J., and C. L. Slayman. 1980. Metabolic modulation of stoichiometry in a proton pump. Biochim. Biophys. Acta 591:224-233.

186. Webb, M. R., C. Grubmeyer, H. S. Penefsky, and D. R. Trentham. 1980. The stereochemical course of phosphoric residue transfer catalyzed by beef heart mitochondrial ATPase. J. Biol. Chem. 255:11637-11639.

187. Wheeler, T. J., and P. C. Hinkle. 1981. Kinetic properties of the reconstituted glucose transporter from human erythrocytes. J. Biol. Chem. 256:8907-8914.

188. Wikström, M. 1984. Pumping of protons from the mitochon- drial matrix by cytochrome oxidase. Nature (London) 308: 558-560.

189. Wikström, M., and K. Krab. 1978. Redox-linked proton pumps in mitochondria, p. 128-139. In E. Schäfer and M. Klingenberg (ed.), Energy conservation in biological membranes. SpringerVerlag, New York.

190. Wikström, M., and K. Krab. 1979. Proton pumping cytochrome c oxidase. Biochim. Biophys. Acta 549:177-222.

191. Wikström, M., K. Krab, and M. Saraste. 1981. Protontranslocating cytochrome complexes. Annu. Rev. Biochem. 50:623-655.

192. Wilbrandt, W. 1977. The asymmetry of sugar transport in the red cell membrane, p. 204-211. In G. Semenza and E. Carafoli (ed.), Biochemistry of membrane transport. Springer-Verlag, New York.

193. Wilson, D. B. 1978. Cellular transport mechanisms. Annu. Rev. Biochem. 47:933-965.

194. Wolfenden, R. 1969. Transition state analogues for enzyme catalysis. Nature (London) 223:704-705.

195. Wolfenden, R. 1974. Enzyme catalysis: conflicting requirements of substrate access and transition state affinity. Mol. Cell. Biochem. 3:207-211.

196. Wyman, J. 1975. The turning wheel: a study in steady states. Proc. Natl. Acad. Sci. U.S.A. 72:3983-3987. 